\title{
Benchmarking sobre Manufactura Esbelta (Lean Manufacturing) en el sector de la confección en la ciudad de Medellín, Colombia
}

\author{
Benchmarking about Lean Manufacturing in \\ the Textile Sector in Medellin
}

\author{
Juan Gregorio Arrieta Posada ${ }^{1}$ \\ Victoria Eugenia Botero Herrera ${ }^{2}$ \\ María Jimena Romano Martínez ${ }^{3}$
}

\begin{abstract}
Resumen
En este documento se presentan los resultados de un benchmarking entre diferentes empresas del sector de la confección en el que se busca evaluar el grado de implementación de la Manufactura Esbelta (Lean Manufacturing) en sus respectivos procesos productivos. Específicamente se trabaja con empresas que fabrican blue jeans, camisas tipo polo y camisetas $t$-shirts. En efecto, este sector industrial en la ciudad de Medellín está muy desarrollado y es uno de los más dinámicos de la industria, por lo tanto es de mucho interés su evaluación.

Para el desarrollo del benchmarking se construyó un cuestionario y se aplicó en las diferentes empresas entrevistadas. El resultado más significativo que se halló es que la implementación de las técnicas de Manufactura Esbelta no se encuentra muy difundida entre las compañías del sector y solamente las que tienen trayectoria de trabajo como empresas exportadoras o licenciatarias de marcas internacionales son las más avanzadas en su aplicación y desarrollo.
\end{abstract}

Palabras clave: Benchmarking, Lean Manufacturing, indicadores de gestión, confección, blue jeans, camisas, camisetas, Manufactura Esbelta.

\begin{abstract}
This document shows the results of a benchmarking study among different firms from the clothing industry that seeks to evaluate their degree of implementation in Lean Manufacturing during their respective productive processes. The study mainly targets firms that produce blue jeans, cotton shirts and t-shirts. Indeed, this industrial sector in the city of Medellin is highly developed and one of the most dynamic, thus the interest to perform an evaluation in this area.

To achieve the benchmarking, a questionnaire was designed and applied to the different firms that were visited. The most significant result obtained was that Lean Manufacturing techniques were not prevalent within the general public. Only those companies with years in the business, exporters or foreign trademarks licensees, were knowledgeable of their application and development.
\end{abstract}

Keywords: Benchmarking, Lean Manufacturing, managing indicators, clothing industry, blue jeans, shirts, t-shirts

1. Profesor Departamento de Ingeniería de Producción, Universidad EAFIT, Medellín-Colombia $<$ jarrieta@eafit.edu.co $>$.

2. Ingeniera de Producción. Universidad EAFIT, Medellín-Colombia. <vboteroh@eafit.edu.co $>$.

3. Ingeniera de Producción. Universidad EAFIT, Medellín-Colombia.<mromanom@eafit.edu.co>. 


\section{INTRODUCCIÓN}

En los últimos 25 años han surgido gran cantidad de estrategias para el mejoramiento de los procesos productivos desde el TQM (Total Quality Management), pasando por el BPR (Business Process Reengineering), y llegando hasta las ventajas competitivas de Porter. Recientemente han surgido las técnicas de Manufactura Esbelta con las cuales se busca la excelencia industrial (Bilalis, Alvizos, Tsironis, \& Van Wasseenhove, 2007).

La Manufactura Esbelta, conocida en inglés como Lean Manufacturing, consiste en la aplicación sistemática y habitual de diferentes técnicas para el mejoramiento de los procesos productivos (Arrieta, 2007). Entre ellas se encuentran las siguientes:

1. Las $5 \mathrm{~s}$ : técnica utilizada para el mejoramiento de las condiciones del trabajo de la empresa. Aquí se desarrollan diferentes pasos orientados hacia el logro de una excelente organización, orden y limpieza en el puesto de trabajo.

2. Los sistemas SMED: técnica empleada para la disminución de los tiempos de cambio de referencia.

3. Los sistemas Poka Yoke: técnica empleada para disminuir los errores en el lugar de trabajo.

4. La administración visual: técnica empleada para presentar visualmente y al alcance de todo el personal los indicadores de desempeño de la empresa.

5. Los grupos Kaizen: técnica que busca el mejoramiento permanente mediante el aporte de ideas de las personas involucradas.

6. Los procesos de mejoramiento basados en 6 sigma: técnica que busca obtener reducir la tasa de defectos menor a un defecto por cada millón de unidades fabricadas.

7. El desarrollo de células de manufactura: técnica que consiste en la implementación de nuevos flujos de producción en la empresa para fabricar artículos con mayor celeridad.

8. Los sistemas TPM: consiste en la implementación del mantenimiento productivo total, para disminuir el tiempo de paro de las máquinas.
9. El análisis de valor del proceso: (Value Stream Mapping): técnica que se aplica para detectar en qué punto del sistema productivo se presentan los mayores desperdicios durante el proceso.

La Manufactura Esbelta busca, además de reducir los "desperdicios" - definidos como aquellos procesos o actividades que no agregan valor al producto (Imai, 1986)-, evaluar y mejorar diferentes indicadores de gestión, tales como el tiempo de entrega, la rotación del inventario, la calidad de los productos, los volúmenes de inventario, la capacidad de los equipos, los costos directos e indirectos de producción, el cumplimiento de pedidos y programas de producción, la participación del personal en los procesos de mejoramiento, el tiempo de desarrollo del producto y los tiempos de espera, entre otros. Todos ellos son indicadores que se pueden medir y aplicar a cualquier sistema productivo.

La Manufactura Esbelta es la base fundamental para la implementación y el éxito de los sistemas en las empresas; es una estrategia administrativa que permite la generación de valor mientras se reducen los desperdicios (Womack, 1996). Es por esto que dichas técnicas de trabajo han adquirido cada vez mayor importancia entre los directivos de empresas para el desarrollo, aplicación y logro de operaciones de clase mundial en sus compañías para asegurar su competitividad en un mercado cada vez más globalizado (Collins, Cordon, \& Julien 1996). En la actualidad, las firmas requieren mejorar permanentemente sus procesos productivos. Una herramienta importante para lograr este objetivo es compararse con otras empresas, ya sean del mismo sector o de otros sectores, para evaluar cómo se encuentran con relación a la implementación de técnicas de mejoramiento de procesos productivos.

La idea de desarrollar este trabajo, que consiste en la aplicación de un cuestionario de 40 preguntas a 30 empresas del sector confección, surge de la inquietud de la academia en evaluar la aplicación de las diferentes técnicas de Manufactura Esbelta en varios sectores productivos de la industria colombiana. En este caso, se ha tomado la industria de la confección por ser una de las más significativas en el país, cuyo polo industrial más importante es la ciudad de Medellín, Colombia. 
En este proyecto se busca determinar la situación del sector de la confección en dicha ciudad en cuanto a la aplicación de la técnica de mejoramiento de procesos productivos conocida como la Manufactura Esbelta (Lean Manufacturing). Ello se realizará mediante el desarrollo de un benchmarking entre diferentes empresas del sector. Aquí se evaluarán las prácticas desarrolladas, así como las diferentes actividades que dichas empresas deben presentar para ser consideradas como firmas que aplican la técnica mencionada.

Este estudio en el sector de la confección dará la base comparativa para determinar el estado de una empresa con relación a aquellas que aplican tales prácticas de mejoramiento y son consideradas empresas exitosas en su aplicación. El resultado permitirá entregarle a la comunidad académica e industrial del sector una herramienta de análisis con la que, respondiendo preguntas muy concretas, facultará evaluar la situación de cualquier firma sobre la aplicación de técnicas de mejoramiento de sus procesos productivos.

En general, muchas empresas se enfrentan a dos situaciones constantes: (a) saber qué camino debe recorrer frente a tanta información disponible sobre el mejoramiento de los procesos productivos; y (b) qué técnicas debe aplicar para el aumento de la calidad, competitividad y productividad. En ese sentido, este documento busca dar claridad sobre qué aspectos se deben evaluar en las empresas si se quiere estudiar la marcha de los procesos empresariales orientados hacia la mejora de los sistemas productivos.

A través de este estudio se verá la importancia y la necesidad de que la alta gerencia observe las técnicas de la Manufactura Esbelta y decida incluirla como una estrategia de competitividad. De esta manera se vincularán los niveles táctico y operativo, incluyendo los indicadores de gestión de la técnica, dentro del sistema de medición de la compañía. Además de considerar la estrategia de manufactura que la empresa ya disponga (costo, calidad, tiempo de entrega y/o flexibilidad), evaluar los indicadores de gestión es una práctica muy importante para generar una cultura de mejoramiento continuo, tal como lo sugieren Martínez y Pérez (2001).
Actualmente, casi toda la industria manufacturera en el mundo ha comenzado a aplicar la Manufactura Esbelta para el mejoramiento de su operación, y Colombia no puede quedarse ajena a dichos cambios. Empresas tales como General Motors-Colmotores, Tetrapak, Unilever Andina y Siemens son ejemplos puntuales de líderes en la implementación de estas técnicas. Estas firmas, ya sea por disposición de las oficinas centrales de la multinacional o simplemente por decisión propia, han comenzado a utilizar diversas herramientas de producción para ordenar y mejorar sus sistemas productivos (Silva, 2003). En la Tabla 1 se señalan algunas empresas líderes en la implementación de herramientas de Manufactura Esbelta en la ciudad de Medellín.

Otras empresas del sector industrial del país, pioneras en el desarrollo e implementación de mejores prácticas dentro de sus operaciones, han adaptado los principios de la Manufactura Esbelta a sus necesidades alcanzando logros y ahorros interesantes. El GEA (Grupo Empresarial Antioqueño) se ha interesado en la filosofía TPM como base de mejoramiento permanente de sus procesos. Para ello ha buscado asesoría en empresas consultoras internacionales líderes en el despliegue de la filosofía como estrategia para su implementación. Actualmente, empresas del GEA, entre ellas la Compañía Nacional de Chocolates y Zenú, se encuentran muy adelantadas en la implementación de la metodología. Cabe también mencionar a la ensambladora de automóviles SofasaRenault, empresa que ha utilizado desde 1991 diversas herramientas de mejoramiento tales como SMED, 5S y TPM. Sofasa-Renault ha logrado el "empoderamiento" de todo su personal, especialmente de sus empleados de planta, en la búsqueda continua de la perfección de sus procesos.

La academia también ha colaborado en el despliegue de las herramientas de mejoramiento que la Manufactura Esbelta ofrece a la industria en general. A través de su vinculación con estudiantes e ingenieros de diversas organizaciones, se ha logrado un gran acercamiento para la implementación de la metodología en todo el conjunto de empresas grandes, medianas y pequeñas del país. Se han desarrollado, además, numerosos cursos, estudios, trabajos de grado e investigaciones como los 
Tabla 1.

Empresas Pioneras en el Desarrollo de Herramientas Lean

\begin{tabular}{|c|c|c|c|c|c|c|c|c|}
\hline \multirow[b]{2}{*}{ Empresa } & \multicolumn{8}{|c|}{ Herramientas } \\
\hline & $5 \mathrm{~S}$ & KAIZEN & TPM & $\begin{array}{c}\text { FABRICA } \\
\text { VISUAL }\end{array}$ & KANBAN & $\begin{array}{l}\text { POKA } \\
\text { YOKE }\end{array}$ & SMED & SIX SIGMA \\
\hline Electroporcelanas Gama & $\mathrm{X}$ & $\mathrm{X}$ & $\mathrm{X}$ & $\mathrm{X}$ & & $\mathrm{X}$ & $\mathrm{X}$ & $\mathrm{X}$ \\
\hline Sofasa & $\mathrm{X}$ & $\mathrm{X}$ & $\mathrm{X}$ & $\mathrm{X}$ & $\mathrm{X}$ & $\mathrm{X}$ & $\mathrm{X}$ & \\
\hline Vestimundo & $\mathrm{X}$ & & & $\mathrm{X}$ & & $\mathrm{X}$ & $\mathrm{X}$ & \\
\hline Grupo Mundial & & & & & & & & $\mathrm{X}$ \\
\hline Colcafe & $\mathrm{X}$ & & $\mathrm{X}$ & & & & & \\
\hline Cía. Nacional de Chocolates & $\mathrm{X}$ & & $\mathrm{X}$ & $\mathrm{X}$ & & & & \\
\hline New Stetic & & & $\mathrm{X}$ & & & & & \\
\hline Noel & $\mathrm{X}$ & & $\mathrm{X}$ & $\mathrm{X}$ & & & & \\
\hline Zenu & $\mathrm{X}$ & & $\mathrm{X}$ & $\mathrm{X}$ & & & & \\
\hline Incolmotos & $\mathrm{X}$ & & $\mathrm{X}$ & $\mathrm{X}$ & & & $\mathrm{X}$ & \\
\hline Grival & & & $\mathrm{X}$ & $\mathrm{X}$ & $\mathrm{X}$ & & & $\mathrm{X}$ \\
\hline Forsa S.A. & & & $\mathrm{X}$ & & $\mathrm{X}$ & & & \\
\hline Cerveceria Unión & $\mathrm{X}$ & $\mathrm{X}$ & & $\mathrm{X}$ & & & & \\
\hline Grupo Corona & $\mathrm{X}$ & $\mathrm{X}$ & $\mathrm{X}$ & & & & & $\mathrm{X}$ \\
\hline Procter \& Gamble & & & $\mathrm{X}$ & $\mathrm{X}$ & & & & \\
\hline
\end{tabular}

Nota. Las x indican la herramienta que las empresas han trabajado más. Aplicar una herramienta no implica no aplicar las otras. (Osorio, Restrepo \& Velandia, 2001).

siguientes: estudios de benchmarking en el sector textil confección en la Universidad Cumhuriyet en Turquía (Ungan, 2007); en el INSEAD, Francia y la Universidad Técnica de Creta, Grecia (Bilalis et al., 2007); en la Universidad de Trieste en Italia (Bolisani \& Scarso, 1996); la Universidad de Delhi en la India (Bheda, Narag \& Singla, 2003); la Universidad de Massachusetts en los Estados Unidos de Norteamérica (Clare \& Mathaisel, 2005). Particularmente, la Universidad Federal de Santa Catarina en Brasil lleva proyectos de benchmarking sobre Manufactura Esbelta en el sector textil, que contó con la asesoría del profesor Dalvio Tubino. El profesor Tubino ha asesorado tesis doctorales y de maestría sobre benchmarking en diferentes sectores, entre las que se encuentran las siguientes:

a) Desarrollo de una herramienta para estudiar el benchmarking made in Brasil (Méndez, 2005); b) Un método de diagnóstico del potencial de aplicación de la Manufactura Esbelta en la industria textil (Pereira, 2006);

c) Desarrollo de un modelo de benchmarking basado en un sistema productivo de clase mundial para evaluación de prácticas y desempeño de la industria exportadora brasilera (Seibel, 2004).

En Colombia, a nivel nacional, se han desarrollado tesis de estudiantes de las facultades de ingeniería industrial, administrativa, de producción, productividad y calidad, entre otras, que estudian sobre la implementación de técnicas de benchmarking. Sin embargo, no se han realizado trabajos específicos al sector de la confección y la aplicación de la Manufactura Esbelta. En tal sentido, la Universidad EAFIT tiene como proyecto futuro el desarrollo de diferentes estudios de benchmarking en diversos sectores industriales de la 
ciudad de Medellín, con el objetivo de brindarles un patrón de comparación que les sirva como herramienta de evaluación y análisis de sus procesos. De igual modo, el CNP (Centro Nacional de Productividad) ha integrado a diversas entidades educativas para ofrecer un entrenamiento en Manufactura Esbelta con un enfoque gerencial y estratégico, dirigido a medianas y grandes empresas que busquen mejorar la calidad de sus procesos ${ }^{1}$.

Un ejemplo de estudios en Manufactura Esbelta se ve en la facultad de Ingeniería Industrial de la Universidad ICESI del Valle del Cauca, en las que se han realizado diversos trabajos sobre el tema, dentro de los cuales se encuentra la investigación de Manotas y Rivera (2007) sobre la medición en Manufactura Esbelta y las relaciones entre actividades y métricas Lean. Los autores integran las formas de medición de la metodología propuestas por diversos académicos buscando consistencia con las etapas y elementos de implementación de la metodología. Asimismo, Osorio, Restrepo y Velandia (2001), de la Universidad EAFIT, realizaron un estudio sobre las mejores prácticas en la manufactura conocidas como herramientas de producción aplicadas al sector metalmecánico de la ciudad de Medellín. En el estudio se realiza un análisis del grado de implementación de algunas de las herramientas Lean en firmas de metalmecánica. Por otro lado, un grupo de importantes empresas de la construcción de Medellín, convocadas por CIDICO (Centro de investigación y desarrollo tecnológico para la industria de la construcción) y con la iniciativa de GESCÓN (Grupo de investigación en gestión de la construcción, Universidad EAFIT), desarrollaron e implementaron un sistema de referenciación para el sector de la construcción (BenchColombia $^{2}$ ) que permite la comparación del desempeño de un proyecto de construcción a nivel local, nacional e internacional, por medio de indicadores de gestión y sus respectivos valores objetivos o benchmark (Botero \& Álvarez, 2006).

1 Centro Nacional de Productividad. $<$ http://www.cnp.org.co/>.

2 Bench-Colombia <http://www.benchcolombia.com/Bench Principal.aspx $>$. Recuperado el 19 de febrero de 2008.
En temas logísticos, existen organizaciones independientes que han realizado procesos de benchmarking. Tal es el caso de la red mundial GS1 ${ }^{3}$, conformada por 105 organizaciones que prestan servicios a diversos sectores industriales y económicos, en más de 150 países. Existe, además, un modelo de referencia de operaciones de la cadena de suministro (SCOR ${ }^{4}$ : Supply Chain Operations Referente Model) desarrollado por el Supply-Chain Council como una herramienta estándar para diagnosticar la gestión de la cadena de suministro. El modelo SCOR permite, mediante la utilización de una estructura determinada de procesos, describir cadenas de suministro utilizando un conjunto común de definiciones.

Por todo lo dicho, se puede observar que existen estudios de benchmarking aplicados a diferentes áreas; sin embargo, el sector confección no registra estudios aplicados a la evaluación de la Implementación de la Manufactura Esbelta. Como información adicional, R. E. Goodman (2002) ha desarrollado una herramienta para valorar rápidamente las empresas con relación a Lean Manufacturing. Dicho documento es el cuestionario RPA en el que a través de respuestas afirmativas y negativas se obtiene una visión general para determinar si la planta tiene cultura Lean o no. Incidentalmente, es importante señalar que Womack, Jones y Ross (1996) mencionan que las tareas de benchmarking entre las empresas son inútiles y se convierten en una perdida de tiempo a menos que se usen para pérsuadir a gerentes reacios a aceptar los temas de mejoramiento usando las técnicas de Manufactura Esbelta.

\section{Sector de la Confección}

Se entiende como sector confección aquel que transforma (diseño, corte y costura) tela, cuero, pieles y otros materiales en prendas listas para ser usadas como indumentaria por el consumidor final (excepto calzado). Esta industria también incluye la fabricación de som-

3 GS1 <http://www.gs1co.org/>. Recuperado el 29 de mayo de 2008.

4 Navactiva <http:/www.navactiva.com/web/es/alog/doc/ informes/2006/03/36349.php $>$. Recuperado el 29 de mayo de 2008 . 
breros, adornos y accesorios (Romero et al., 2000). La industria de la confección es global; todo ser humano necesita vestirse. Es una industria intensiva en mano de obra, y en los últimos años ha migrado de los países desarrollados a los países en vía de desarrollo. Sin embargo, estos últimos requieren mejorar y evaluar sus procesos productivos con el propósito de permanecer competitivos y garantizar su desarrollo económico (Bheda et al., 2003).

En el mundo de hoy, la industria textil y de confección hace una gran contribución a las economías nacionales, especialmente en los países emergentes. Una gran cantidad de estos países están explotando el sector en beneficio de su propio crecimiento económico (Dickerson, 1995). En este contexto, Colombia es reconocida internacionalmente como un país que presenta grandes fortalezas en el negocio de los textiles y las confecciones, en particular el de la moda. Sin embargo, algunos subsectores de textiles y de confecciones han decrecido durante el período enero-junio de 2009, entre ellos se pueden nombrar a los subsectores de hilados, tejidos, tejido de punto, tejido plano y ropa de hogar.

Desde principios del siglo XX, las principales industrias textiles surgieron en Colombia, especialmente en la región antioqueña, distribuidas en municipios como Medellín, Bello e Itaguí. A través de la historia, el país fue desarrollando su infraestructura como cultivador y exportador de algodón de longitud media y corta en regiones del Atlántico, César, Meta, Valle y Tolima. El fortalecimiento algodonero permitió el desarrollo de la industria textil de Antioquia y Manizales. Hacia 1907, se construyeron en esta ciudad las dos primeras grandes fábricas, Coltejer y la Compañía Antioqueña de Hilados y Tejidos. Al presente, esta última hoy forma parte de Fabricato, otra de las principales firmas textiles colombianas. Estas empresas comenzaron a crear nuevas fuentes de empleo, así como la creación y adecuación de nuevas tecnologías. Durante los últimos años, ambas empresas han manufacturado tejidos (driles, índigos, popelinas y corduroys de la más alta calidad) así como géneros y gabardinas en algodón para el mercado interno y a los mercados latinoamericanos, norteamericanos y europeos.
Por la versatilidad y la calidad en su mano de obra, la ciudad de Medellín es líder manufacturero en la producción de ropa para los segmentos masculino, femenino, juvenil e infantil. Igualmente es reconocida por la confección de prendas casuales y formales, vestidos de baño, ropa interior, ropa deportiva y jeans, que en muchas ocasiones son fabricados para grandes marcas que trabajan bajo la modalidad de maquila o paquete completo. En la actualidad, Medellín es el centro del sector confección en Colombia. Sin embargo, recientemente otras regiones como Bogotá/Cundinamarca y el Atlántico han empezado a emerger en el sector con una fuerte presencia. Un ejemplo es la composición del Cluster Textil/ Confección Diseño y Moda de Medellín/Antioquia. Es relevante indicar que el conglomerado está constituido por microempresas $(90.4 \%)$, pequeñas empresas $(7.2 \%)$, medianas empresas $(1.9 \%)$, y grandes empresas $(0.5 \%)$ (recuperado de $<$ ww.inexmoda.org.co $>$ ).

Los más importantes productos de confección exportados son pantalones largos, pantalones con peto; pantalones cortos (calzones) y shorts de tejidos, llamados mezclilla o denim, para hombres o niños; tejidos de punto de ancho superior a $30 \mathrm{~cm}$, con un contenido de hilados de elastómeros superior o igual a $5 \%$ en peso; t-shirts y camisetas interiores de punto, de algodón, sostenes (corpiños), pantalones largos, pantalones con peto, pantalones cortos (calzones) y shorts de algodón para mujeres o niñas, excepto los de punto. Los principales destinos de exportación durante el año 2008 fueron Venezuela (65.13\%), Ecuador (10.12\%), México (5.13\%), Perú (3.60\%) y Estados Unidos (2.91\%) (recuperado de www.inexmoda.org.co).

El sector confección colombiano es una de las industrias clave de la nación y forman parte del sector textil-confección, responsable del $9 \%$ del PIB productivo del país, $24 \%$ del empleo en manufactura y $7 \%$ del total de las exportaciones 5 . El gobierno colombiano implementó reformas económicas a principios de los $90 \mathrm{~s}$ para abrir la economía del país a la inversión extranjera reduciendo los aranceles. Actualmente, el sector recibe

5 Inexmoda, Instituto para la Exportación de la Moda <http:// www.inexmoda.org.co/>. Recuperado el 13 de febrero, 2008. 
los beneficios de la extensión del Atpdea hasta junio del 2010 y se encuentra a la espera del TLC con Estados Unidos. Las reformas anteriormente mencionadas y la ubicación estratégica de Colombia ayudaron a que las exportaciones del sector incrementaran $32.5 \%$ entre enero y octubre del 2007. Venezuela fue el principal destino, con ventas de 116,24 millones de dólares en el mismo período ${ }^{6}$.

El sector textil-confección colombiano está compuesto por cerca de 450 fábricas de textiles y 1,200 fábricas de confecciones con más de 20 trabajadores en cada una de ellas. Emplea a 200,000 personas directas y 600,000 indirectas, aportando el $24 \%$ del empleo total manufacturero de Colombia. Estas empresas están ubicadas en siete ciudades del país, principalmente en Medellín, que representa el 53\% de la producción textil del país y el $35 \%$ de la producción de prendas de vestir?

\section{Tipos de Industrias dentro del Sector de la Confección}

El sector de la confección en Colombia no está necesariamente integrado a lo largo de su cadena de producción, sino que existen diferentes tipos de empresas dentro del sector. De acuerdo con la etapa de producción en que se encuentran, las empresas se dividen en la siguiente clasificación:

- Maquila: son empresas ensambladoras que realizan un proceso de confección a terceros, mediante un compromiso entre la empresa que realiza el trabajo y el dueño de la producción o de la marca. Se caracterizan porque utilizan insumos y tecnología generalmente importados; emplean mano de obra local para dar una terminación a los productos que luego se reexportan. Este tipo de manufactura ha ido

6 Ministerio de Comercio, Industria y Turismo. $<$ http://www. mincomercio.gov.co/eContent/home.asp $>$. Recuperado el 13 de febrero, 2008.

$7<$ (http://inexmoda2.private.arkix.com/TextilConfecci $\% \mathrm{C} 3 \% \mathrm{~B} 3 \mathrm{n} /$ ElsectorTextilydelaConfeci $\% \mathrm{C} 3 \% \mathrm{~B} 3 \mathrm{nColombi}$ ano/tabid/1105/Default.aspx $>$. Recuperada el 22 de febrero, 2008. disminuyendo poco a poco en la industria nacional y se está reemplazando con la industria del paquete completo.

- Paquete completo: Empresas cuyo proceso productivo comprende todos los pasos en la cadena de la producción de las prendas de vestir. Los pasos incluyen desde la producción de la tela, los insumos, la confección y el diseño total del producto hasta sus ventas y trámites de exportación. En este caso, ni el producto ni la marca son propiedad de la empresa, y un tercero autoriza a la empresa a fabricarlo bajo la modalidad de paquete completo.

- Marca propia: Distintivo que asocia un determinado bien o servicio con el agente económico que lo produce o comercializa. Este puede ser el nombre o razón social de una empresa, una palabra, una etiqueta o cualquier otro símbolo que lo identifique. De acuerdo con esta estructura, la empresa es propietaria del producto y su marca, de manera que lo puede fabricar y comercializar sin problemas.

- Licencia: Esta figura es usada por las multinacionales de ropa, como en el caso Levi's. En esta modalidad, la empresa internacional negocia con una empresa local el uso de su marca. La empresa local diseña, selecciona las materias primas, insumos, produce las prendas, comercializa y distribuye. Eventualmente, la casa matriz puede dar licencia para que la empresa nacional comercialice las prendas con su marca en diferentes países. Obviamente, la empresa nacional aprovecha la popularidad de la marca internacional. En Colombia, Levi's trabaja la modalidad de licencia con C.I. Expoforo y la de paquete completo con C. I. Jeans.

En el año 2008, el sector textil-confección en Colombia tuvo una crisis, que impactó con una reducción del $4 \%$ en el consumo de confecciones y pérdidas económicas de US\$80 millones. La principal causa fue la recesión de la economía mundial, que estuvo ligada a una contracción de la demanda mundial y a la devaluación del dólar. El contrabando y el lavado de dinero también contribuyeron a la crisis del sector, pues introdujeron mercancía a precios bajos perjudicando el ingreso de miles de familias vinculadas a las empresas textiles o 
de confección. Como resultado de esta situación, varias plantas de producción han cerrado y más de 24,000 trabajadores perdieron sus puestos de trabajo. ${ }^{8}$

En este momento, el sector textil-confección se encuentra en la búsqueda de nuevas oportunidades, pues las exportaciones cayeron entre enero y mayo de 2009 un $23 \%$. Por otro lado, las relaciones con los países vecinos no están en óptimas condiciones en estos momentos, por lo que se hace imprescindible que el sector explore otros mercados. Junto con esta medida, la industria requiere incrementar el consumo interno a través de mayor innovación, tanto en los productos como en los procesos, y buscar una mayor integración en toda la cadena productiva (Revista Dinero, junio de 2009).

Las ventas del sector confección durante el 2008 fueron aproximadamente de 200 millones de dólares entre camisas $t$-shirt y camisas tipo polo, y de $120 \mathrm{mi}$ llones de dólares en blue jeans (Revista La Nota.com, mayo del 2009). Los datos aquí resumidos muestran la gran importancia que tiene el sector para la economía del país, y en especial para la ciudad de Medellín. Por eso es imprescindible que un proyecto con las características planteadas se lleve a cabo, para evaluar cuál es la situación de la industria textil en cuanto a la aplicación y desarrollo de la Manufactura Esbelta. Al mismo tiempo, se debe promover el concepto de la utilización del benchmarking como una herramienta de competitividad y que, a la vez, sirva de apoyo a las etapas que se deben ejecutar para desarrollar procesos de mejora permanente en la industria nacional de la confección.

\section{Hipótesis}

De lo expuesto hasta ahora se puede concretar la siguiente pregunta para la hipótesis central de este trabajo: “¿Es posible medir el nivel de implementación, desarrollo y seguimiento de las técnicas de Manufactura Esbelta y el estado en el cual se encuentran las empresas de

8 Verificar en $<$ http://www.dinero.com/noticias-empleo/empleo-reto-del-sector-textil/51996.aspx $>$. Recabado el 17 de enero, 2008. confección con relación a la aplicación de esta filosofía de mejoramiento de los procesos productivos usando un modelo de benchmarking?" Para responder a esta interrogante se busca validar que sí es posible medir el sector de la confección en la ciudad de Medellín en las empresas que fabrican blue jeans, camisas tipo polo y camisetas interiores, $t$-shirts utilizando un benchmarking. Luego, aplicando los diferentes indicadores de desempeño y las prácticas o técnicas de la Manufactura Esbelta, es factible evaluar el sector objetivo con relación a la aplicación de dicha herramienta de mejoramiento.

\section{INTRODUCCIÓN AL BENCHMARKING Y A LOS MATERIALES, Y MÉTODOS USADOS}

La elaboración de este proyecto comenzó con una investigación teórica sobre Manufactura Esbelta, benchmarking, el sector confección y el desarrollo de cuestionarios para aplicar en las empresas. Adicionalmente, como investigación de campo, se realizaron visitas a las empresas de confección con los cuestionarios mencionados y que se entregaron durante las entrevistas a los directores de producción.

El desarrollo de este proyecto se realiza a través de la metodología de benchmarking sobre Manufactura Esbelta para el sector confección, en el que se toma como base el modelo que M. J. Spendolini propone en su libro Benchmarking (1994). Se aplicó la metodología de cinco pasos propuesta por Spendolini, pues esta describe un modelo genérico; es decir, la metodología abarca todo el conocimiento de las compañías pioneras en el tema, permitiendo la personalización según las necesidades específicas de cada caso.

Las cinco etapas del proceso se grafican en la Figura 1, que se describen brevemente a continuación:

1. Determinar a qué se le va a aplicar el benchmarking: aquí se identifican los clientes y sus necesidades, y definir los procesos a los cuales se les va a efectuar el benchmarking. 


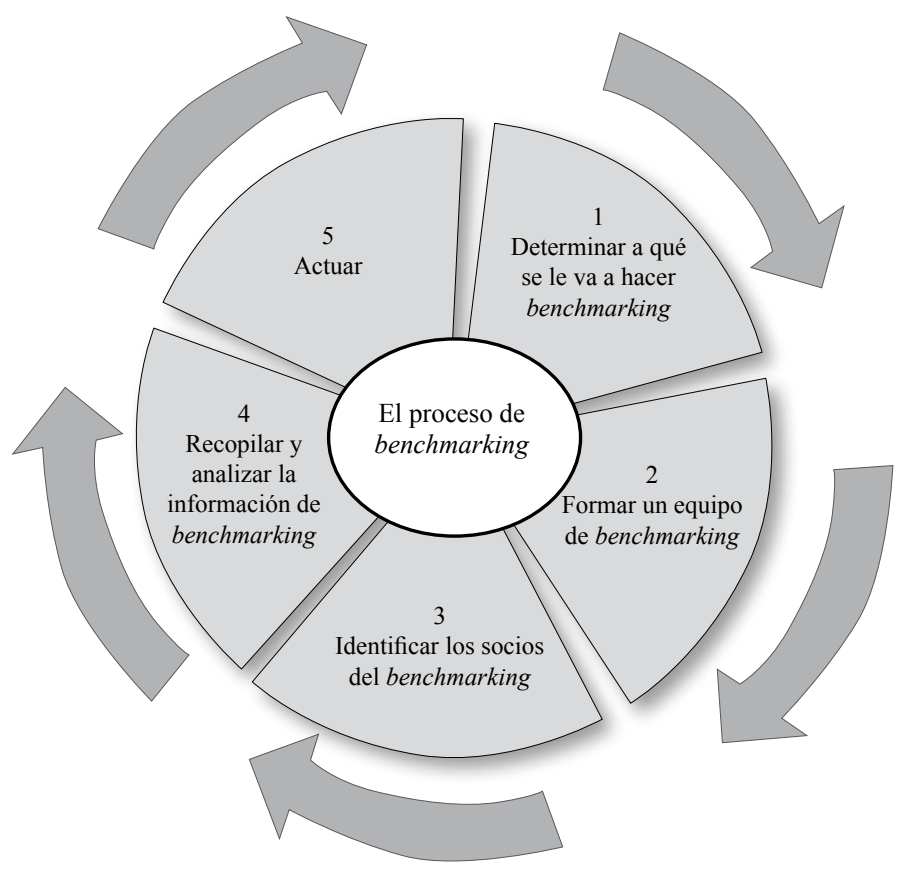

Figura 1. El proceso de benchmarking según M. J. Spendolini

2. Formar un equipo de benchmarking: se escoge el equipo y se señalan las responsabilidades de cada miembro.

3. Identificar los socios de benchmarking: en esta etapa se identifican las fuentes de información y las mejores prácticas.

4. Recopilar y analizar la información: seleccionar los métodos de recolección de información, recopilar y resumir para hacer el análisis.

5. Actuar: se hacen las recomendaciones según las necesidades del cliente. En este paso se incluye el seguimiento que el proceso necesite.

A continuación se describirán cada uno de los pasos del proceso, en donde se explicará de manera concisa el enfoque dado por Spendolini; luego, se hará un acercamiento a la filosofía Lean y al sector en estudio.

\section{Determinar a qué se le va a hacer benchmarking}

En este primer paso de la metodología del benchmarking, el objetivo principal es encontrar los clientes o usuarios finales de la información y la definición clara y específica de sus necesidades críticas. Spendolini afirma que para obtener un proceso de benchmarking exitoso es de vital importancia ser muy específico con la información y las necesidades de los clientes, ya que de esta forma no se pierde tiempo ni se malgastan recursos.

Las empresas de la ciudad de Medellín seleccionadas para hacer el proceso de benchmarking en Lean Manufacturing son 30 reconocidas firmas del sector de la confección, de tamaño representativo. Todas ellas manufacturan diferentes productos entre los que ofrecen blue jeans, camisetas ( $t$-shirts) y camisas tipo polo. Dichas empresas trabajan bajo diferentes estructuras productivas: unas producen bajo marca propia; otras por paquete completo; otras son totalmente maquilas. La selección de estas empresas se hizo de acuerdo con la información financiera presentada en el libro La Nota Económica Estudio Platinum (2006). Aquí se presenta de manera muy concreta el tipo de empresa de confección, y los diferentes indicadores de gestión que dan mayor información sobre cada compañía. Adicionalmente, se realizaron entrevistas a ejecutivos de empresas recomendadas por las aquellas visitadas 
inicialmente, así como compañías que también forman parte de la cadena productiva y las que abastecen procesos de apoyo (empresas estampadoras, lavanderías, entre otras).

\section{Escoger el equipo y las responsabilidades} de cada uno

El equipo de trabajo Manufactura Esbelta está integrado por las siguientes personas de cada una de las plantas y el responsable o analista del benchmarking:

- Director de Mejoramiento Continuo

- Director de Planeación

- Director de Calidad

- Director de Producción

\section{- Analista de Benchmarking}

Para tener un mejor resultado de las entrevistas e información que se obtiene del equipo de benchmarking, lo más adecuado es obtener los datos de todas las personas del grupo. Sin embargo, si no es posible tenerlos a todos, la entrevista con el Director de Planeación o de Producción es suficiente. Las tareas de cada uno de los miembros del equipo de benchmarking buscan principalmente la obtención de la información y entregarla al analista y su equipo de trabajo. A su vez, la función de los analistas es la de aplicar el mecanismo de obtención de la información, realizar las entrevistas con el personal de la empresa, explicar lo que no se entienda bien; luego, se abocan a desarrollar los análisis y la presentación de los resultados.

\section{Recopilar, analizar la información de benchmarking y actuar}

En esta parte, el equipo aplica el mecanismo de evaluación, que se realiza a través del desarrollo de cuestionarios impresos o virtuales, con entrevistas personales o telefónicas. Sin embargo, es preferible que toda la información se obtenga personalmente, para asegurar una buena comunicación entre el equipo de análisis y los entrevistados. Esto facilita el trabajo, pues, en caso de necesitarse aclaraciones, estas se hacen en el momento.
El cuestionario que se aplica está compuesto por 40 preguntas enfocadas a validar el cumplimiento de los siguientes indicadores:

1. LMPF (Lean Manufacturing Piso de la Fábrica): Observa el piso de la fábrica; es decir, se evalúan principalmente los procesos productivos y sus controles.

2. LMPP (Lean Manufacturing Personal de Planta): Determina el personal operativo de la empresa evaluando principalmente si existe algún grado de participación del personal en las tareas de mejoramiento continuo de la empresa.

3. LMDD (Lean Manufacturing Diseño del Producto): Indica el diseño del producto. En este indicador se prueba si existen criterios de ingeniería concurrente para el diseños de los diferentes productos

4. LMAC (Lean Manufacturing Administración Cadena de Abastecimiento): Indica la cadena de abastecimiento. Aquí se evalúa principalmente si la empresa tiene algún tipo de intercambio electrónico de información con sus clientes y proveedores.

A continuación se presentan los indicadores pormenorizados que se evalúan en cada grupo de preguntas:

a) LMPF1: Número de partes en común entre los productos

b) LMPF2: Rotación del inventario

c) LMPF3: Tiempo de entrega (Lead Time) de las ordenes a los clientes

d) LMPF4: Porcentaje de entregas justo a tiempo realizadas por los proveedores de la empresa

e) LMPF5:Capacidad de respuesta a los cambios en la demanda

f) LMPF6: Porcentaje de partes entregadas a tiempo entre las diferentes secciones en la línea de producción.

g) LMPF7: Empresa evalúa el costo de los desperdicios (COW: Cost of Waste Computation)

h) LMPF8: Flexibilidad en la producción 
i) LMPF9: Uso de las tarjetas kanban para las actividades de programación de la producción

j) LMPF10: Implementación de células de manufactura

k) LMPF11: Aplicación de técnicas de balanceo de líneas

1) LMPF12: Estandarización de los procesos productivos de la planta

m) LMPF13: Implementación de la fábrica visual

n) LMPF14: Cambio rápido de herramientas

o) LMPF15: Implementación del mantenimiento productivo total

p) LMPF16: Aplicación del control estadístico de la calidad en productos y procesos productivos

q) LMPF17: Implementación de sistemas Poka Yoke en la empresa.

r) LMPF18: Instalaciones de la planta son limpias, seguras, ordenadas, iluminadas, los niveles de ruido son bajos y el aire es de buena calidad

a) LMPP1: Número de sugerencias realizadas por empleados al año

b) LMPP2: Número de sugerencias realizadas por empleados que se implementan al año

c) LMPP3: Eventos o talleres Kaizen realizados

d) LMPP4: Grado de polivalencia de los operarios de la planta

e) LMPP5: Cantidad de horas de entrenamiento por empleado por año en temas de Lean Manufacturing

f) LMPP6: Indicador del porcentaje de trabajadores que conocen el proceso Lean Manufacturing $\mathrm{y}$ tienen una visión general del mismo

g) LMPP7: Capacidad de la parte operativa para detener la planta (polivalencia)

h) LMPP8: Planes de reconocimiento sobre ideas de mejoramiento implementadas i) LMPP9: Aplicación de las $5 \mathrm{~s}$ en sus procesos productivos

j) LMPP10: Indicador para evaluar la ausencia de barreras entre directivos y operarios

a) LMDD1: Indicador para evaluar la aplicación de la ingeniería concurrente o simultánea

b) LMDD2: Indicador de establecimiento de parámetros del diseño de productos

c) LMDD3: Indicador de porcentaje interno de defectos

d) LMDD4: Indicador de porcentaje de productos sobrantes

e) LMDD5: Indicador de productos que se fabrican usando módulos de confección

f) LMDD6: Tiempo de entrega (Lead Time) del desarrollo de un producto nuevo

a) LMAC1: Indicador de partes diseñadas en conjunto con los proveedores

b) LMAC2: Porcentaje de documentos intercambiados con los proveedores en que se usa EDI

c) LMAC3: Indicador de la cantidad de proveedores certificados con los que la empresa trabaja

d) LMAC4: Cantidad de proveedores con acceso al sistema de planeación y control de la producción de la compañía

e) LMAC5: Cantidad de proveedores a los que la empresa tiene acceso a su sistema de planeación y control de la producción

f) LMAC6: Uso del conocimiento y experiencia del proveedor para hacer mejoras en sus procesos productivos

En la metodología, actuar se refiere al análisis de la información, la construcción y la presentación de resultados, generalmente acompañados con sugerencias para mejoras que se habrían detectado mediante el estudio realizado. Sin embargo, esta etapa no se desarrollará en el presente trabajo pues no es su objetivo. 


\section{METODOLOGÍA EMPLEADA}

Para realizar el benchmarking sobre Manufactura Esbelta en el sector de la confección, el procedimiento es dividir la empresa en diferentes áreas de evaluación y analizar los indicadores de gestión que se relacionan con ellas. La selección de las áreas se hace mediante los diversos indicadores de gestión; cada área es evaluada por diferentes indicadores y se determinarían cómo estos indicadores participan en el desarrollo y trabajo de las herramientas de Manufactura Esbelta. Se tienen dos tipos de indicadores: a) los que representan el Desempeño, que son aquellos que se asocian directamente a un cálculo o fórmula para la medición; y, b) los indicadores relativos a las Prácticas de Gestión (herramientas), los cuales hacen referencia a si la empresa tiene o no implementadas las herramientas que se usan en la Manufactura Esbelta.

Según se aprecia en los cuadrantes de la Figura 2, el eje horizontal grafica la calificación promedio obtenida para los indicadores clasificados en "herramienta", y el eje vertical los de "desempeño". Los ejes de origen están ubicados en $60 \%$ respectivamente, porque es el valor mínimo esperado y tolerable. En efecto, una empresa que esté trabajando en las primeras etapas de
Manufactura Esbelta debe como mínimo cumplir con el $60 \%$ de los indicadores (Seibel, 2004).

Las empresas cuyos resultados se encuentren en el cuadrante I, y especialmente las ubicadas hacia la esquina superior derecha (coordenada 100,100), demuestran una posición muy buena con relación a la aplicación de la Manufactura Esbelta. Inversamente, las posicionadas en el cuadrante III, especialmente si están ubicada hacia la esquina inferior izquierda (coordenada 0,0 ), observan pobres condiciones de trabajo en consonancia con las técnicas. Por otro lado, las empresas cuyos resultados estén por debajo del $60 \%$, es decir las posicionadas entre los cuadrantes II y IV, son aquellas que tienen oportunidad de mejora y por lo tanto requieren plantear opciones de mejoramiento dentro de la compañía.

Si alguna empresa se encuentra en el cuadrante IV, dicha posición indica que la gestión gerencial está aplicando bien las herramientas de Manufactura Esbelta; no obstante, le falta mayor definición y desarrollo en los indicadores de desempeño, indicadores que les permiten evaluar su aplicación. La empresa que se encuentre en el cuadrante II demuestra que tiene indicadores de gestión medibles y evaluables sobre diversos aspectos consi-

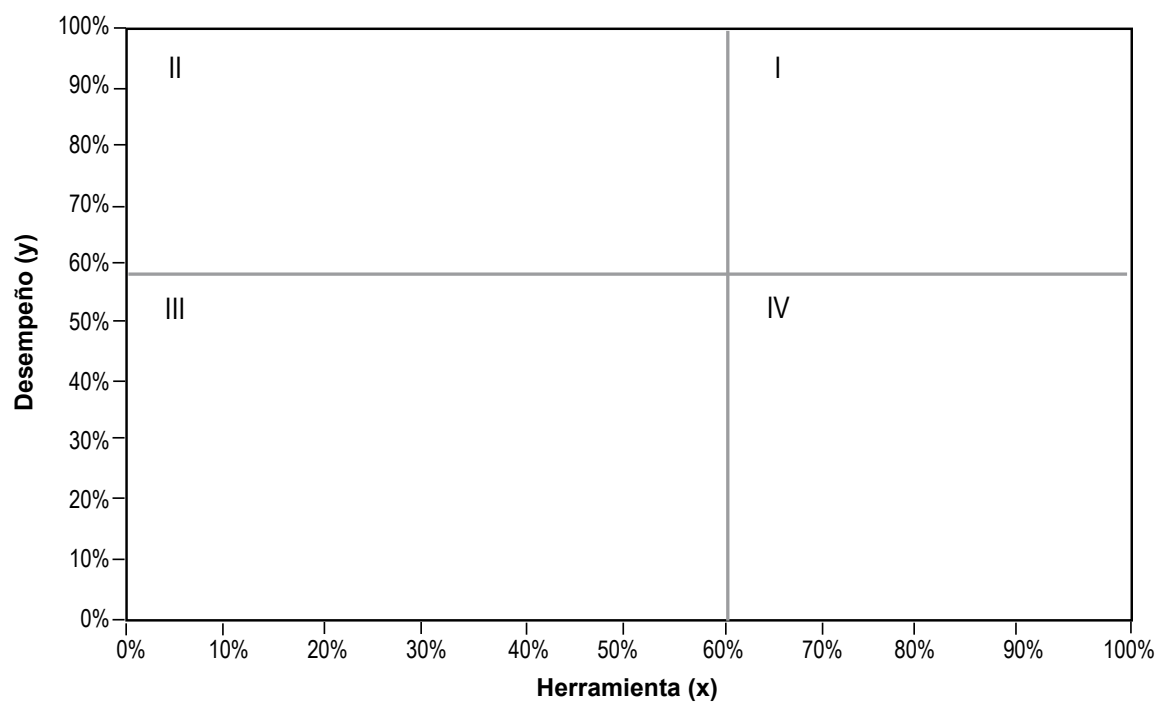

Figura 2. Cuadrante 
derados en la Manufactura Esbelta, pero la aplicación de las herramientas no se encuentra suficientemente desarrollada.

En la Figura 3 se presenta el cuadrante dividido en seis sectores que determinan la calificación de las empresas según su posición relativa de acuerdo con una evaluación cualitativa. Dicha evaluación cataloga a las empresas en: de clase mundial, promisoria, rezagada de contrapeso y vulnerable (Seibel, 2004). A continuación se define cada clasificación:

a) La empresa de clase mundial es aquella que en su calificación, tanto de herramientas como de desempeño, obtiene porcentajes mayores al $80 \%$. Esto significa que tiene una excelencia operacional y presenta condiciones muy favorables para competir en mercados internacionales.

b) La empresa promisoria es aquella que presenta una calificación de entre $60 \%$ y $80 \%$ en herramientas, y en desempeño se encuentra entre un $50 \%$ y $60 \%$, por debajo de lo mínimo deseado. Estas cifras indican que la empresa está trabajando e implementando técnicas de mejoramiento. En este caso los resultados de desempeño todavía no son los más satisfactorios, o aún no los han empezado a eva- luar profundamente. Sin embargo, estas empresas tienen un futuro promisorio si continúan con sus programas de mejora.

c) Las empresas rezagadas, o retardatarias, son aquellas cuya calificación se encuentra por debajo del $50 \%$ en ambos ítems de evaluación. Este tipo de industria es la que mayor trabajo debe invertir para cambiar su tendencia, enfocarse en mejorar y en la aplicación de sistemas de medición de sus procesos productivos.

d) Las empresas llamadas de contrapesos son aquellas que presentan entre $50 \%$ y $60 \%$ de calificación, o quizás menores al mínimo deseado, tanto en herramientas como en desempeño. Este tipo de empresas han aplicado algo de técnicas de mejoramiento; pero, al igual que las rezagadas, deben realizar mucho trabajo para cambiar y mejorar sus procesos productivos y administrativos.

e) Las empresas vulnerables son aquellas que tienen una calificación entre $50 \%$ y $60 \%$ para la aplicación de herramientas, evaluación que está por debajo del mínimo aceptable. También presentan una calificación entre $60 \%$ y $80 \%$ para el desempeño. En estos casos, el tema que preocupa es que no hayan implementado bien las técnicas de mejoramiento de

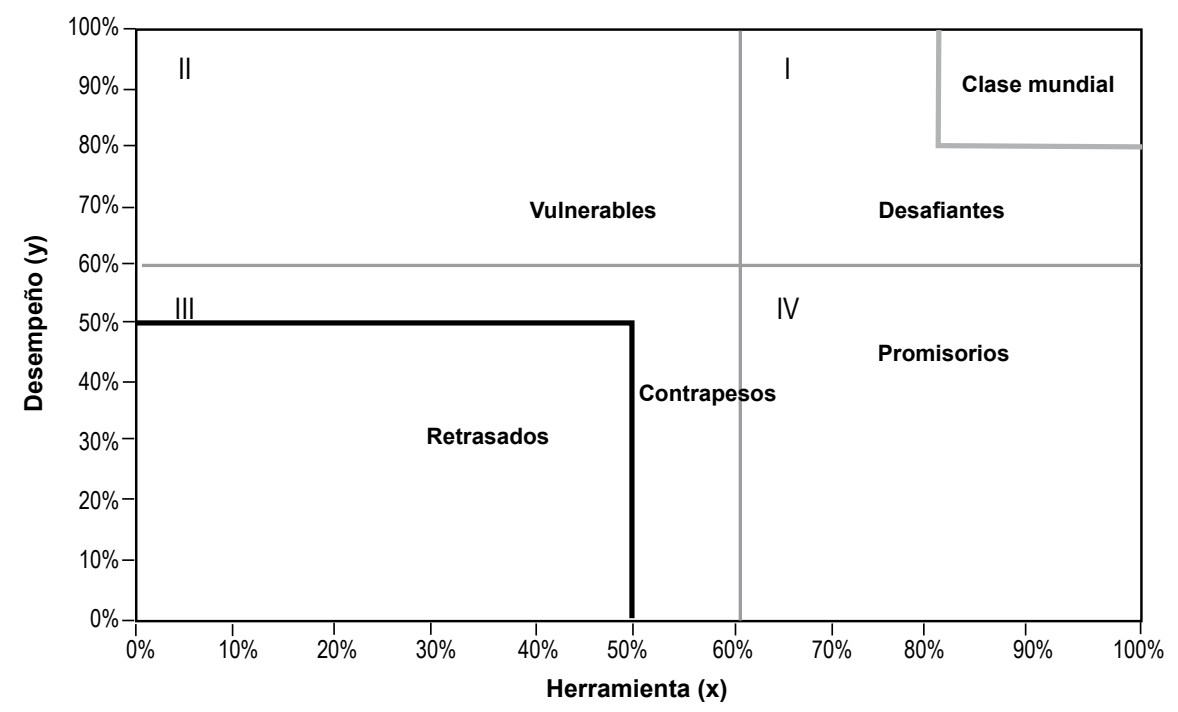

Figura 3. Cuadrante empresas de clase mundial 
procesos que los procedimientos de la Manufactura Esbelta requiere.

\section{RESULTADOS}

Durante la investigación se entrevistó a personal clave de 30 empresas de confección de la ciudad de Medellín, divididas de la siguiente manera: 23 dedicadas a la confección; 3 a la estampación y bordados; 4 a los lavados. Después de aplicar el cuestionario en las diferentes empresas del sector de la confección, los resultados obtenidos indican cuál es el estado en el sector se encuentra.

\section{Descripción general de los resultados} de la muestra

La Figura 4 muestra el promedio general de las 30 empresas contactadas donde se observa que el total de la muestra tiene una calificación del $61.17 \%$. Dicha cifra indica que el sector evaluado está por encima del valor mínimo esperado $(60 \%)$.

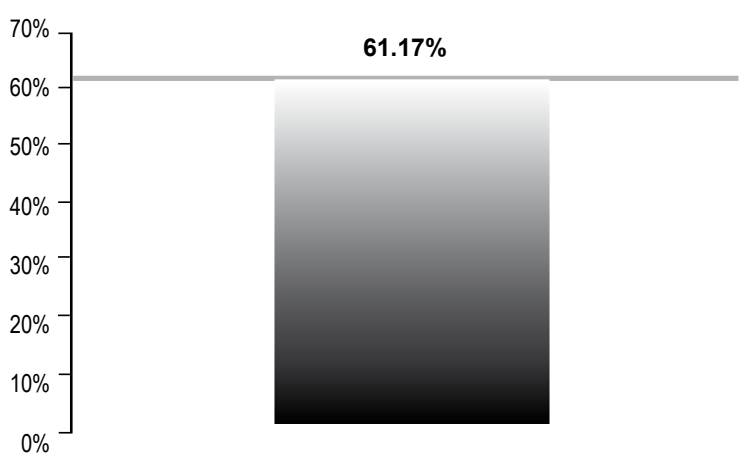

Figura 4. Promedio general de la muestra

El cuadrante de la Figura 5 indica el promedio general del total de la muestra, detallado por herramienta y desempeño.

La Figura 6 presenta cómo se encuentra cada una de las empresas en el cuadrante en el que se evalúa el tipo de compañía con relación a la clase mundial.

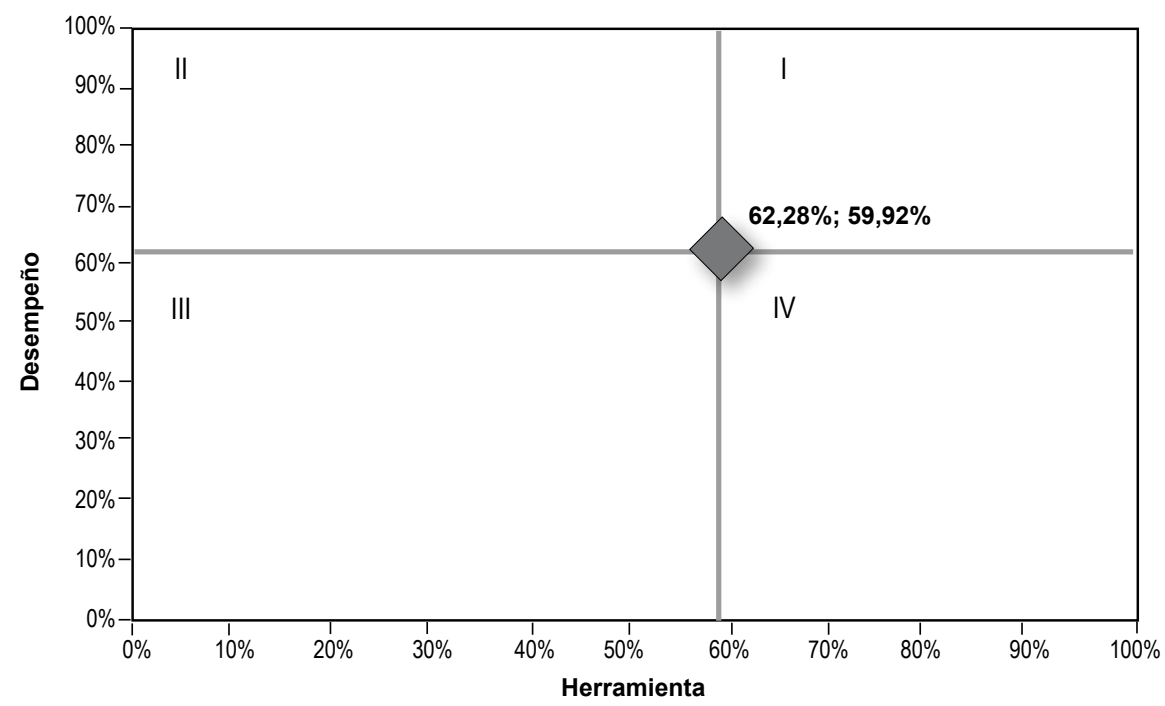

Figura 5. Promedio general de la muestra, detallado por herramienta y desempeño 


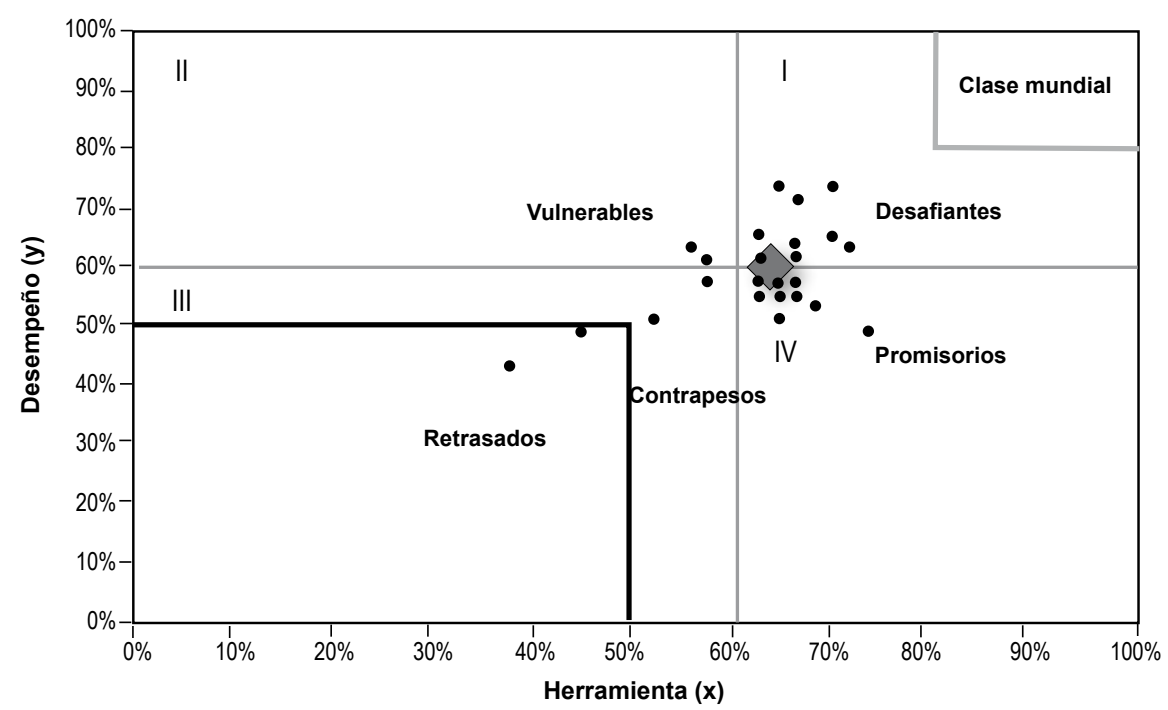

Figura 6. Cuadrante empresas de clase mundial

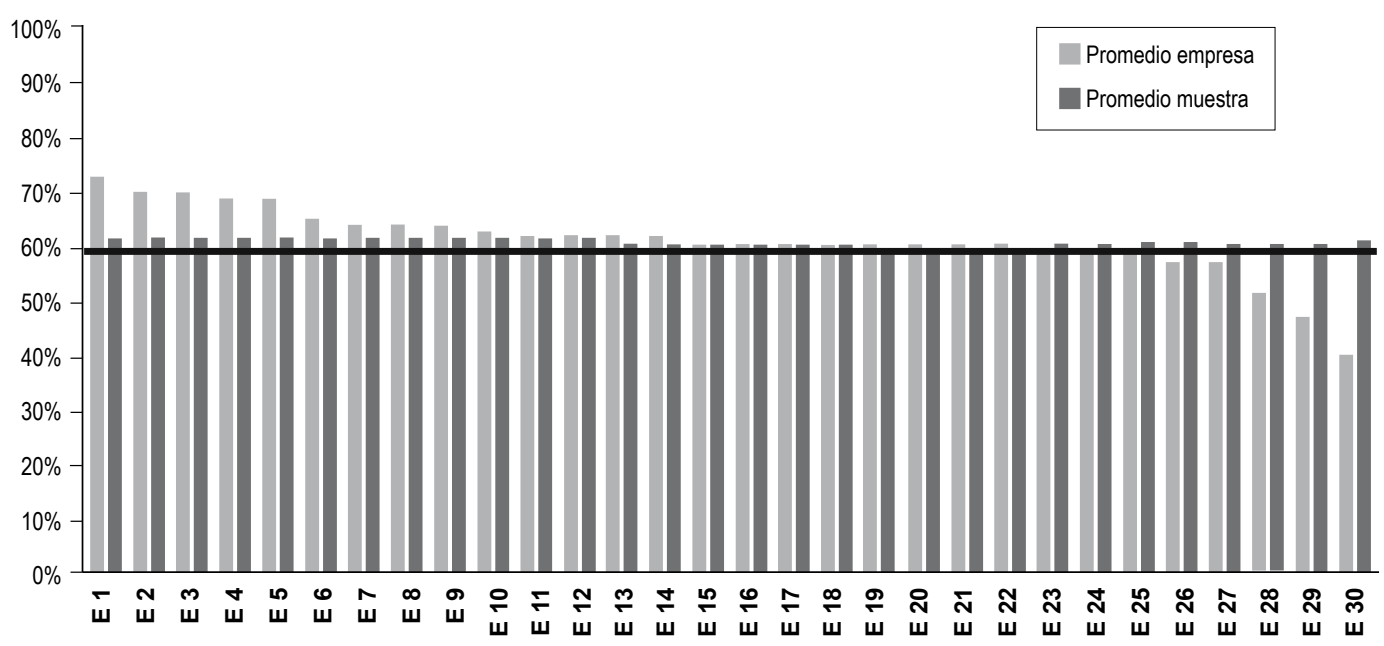

Figura 7. Promedio general de cada empresa vs. promedio general de la muestra vs. nivel esperado

En la Figura 7 se compara la calificación promedio general de cada una de las empresas con el promedio general de la muestra $(61.17 \%)$ y con el nivel mínimo esperado $(60 \%)$.

La Figura 8 presenta en qué percentil se encuentra cada empresa de acuerdo con el promedio obtenido en herramienta o desempeño.
El radar de la Figura 9 detalla y compara el promedio general de cada una de las áreas de trabajo-LMPP (50.40\%), LMPF (66.07\%), LMAC (45.11\%), LMDD $(80.44 \%)-$, con el promedio general de la muestra (61.17\%) y con el nivel mínimo esperado (60\%). 


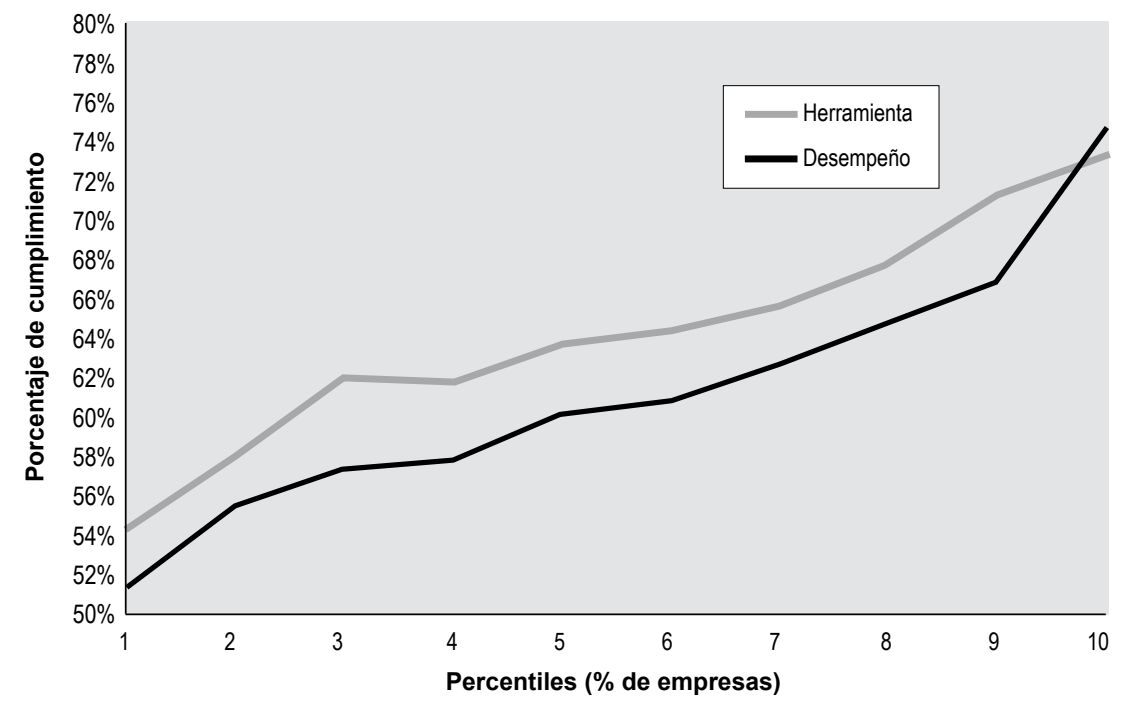

Figura 8. Percentiles por promedio de las empresas del sector

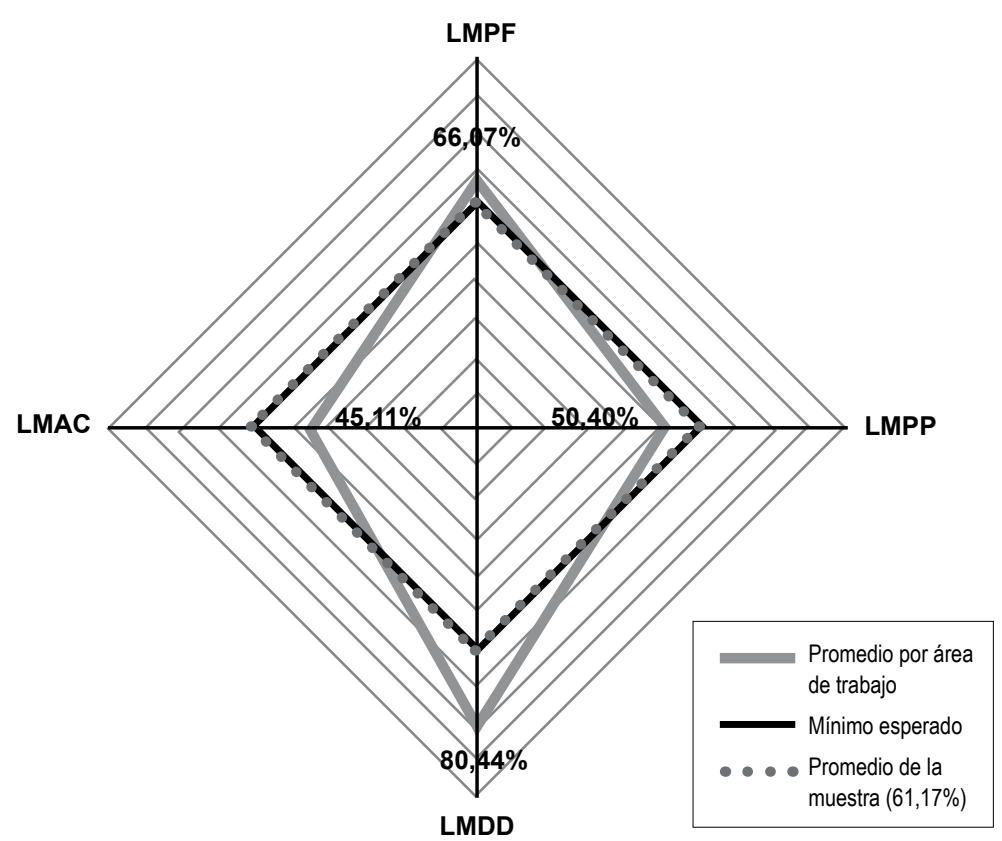

Figura 9. Promedio general detallado por área de trabajo

La Figura 10 muestra el comportamiento de los indicadores para el promedio general de todas las empresas evaluadas.

\section{Descripción por empresas según sus procesos}

Las empresas con procesos de diseño, corte, confección y acabados presentan el mayor promedio (62.5\%), 


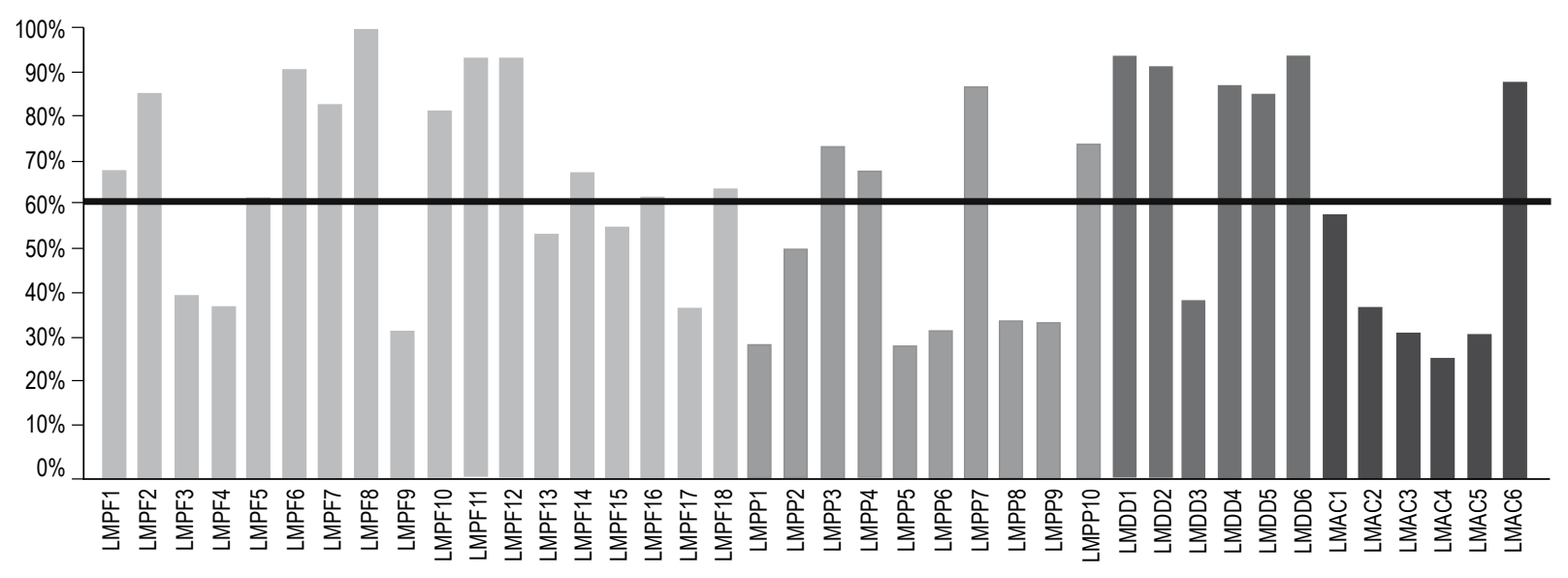

Figura 10. Comportamiento general de cada uno de los indicadores

seguido por las empresas con procesos de acabados (62.14\%). Ambos procesos están por encima del promedio general de la muestra y del nivel esperado. Las empresas con el proceso de diseño, corte y confección $(60.53 \%)$ se encuentran en un nivel inferior al promedio de la muestra, pero superior al $60 \%$.
La Figura 11 muestra el promedio en herramienta y desempeño para cada proceso productivo de las empresas, comparándolo con el promedio general del total de la muestra y el mínimo aceptable (60\%).
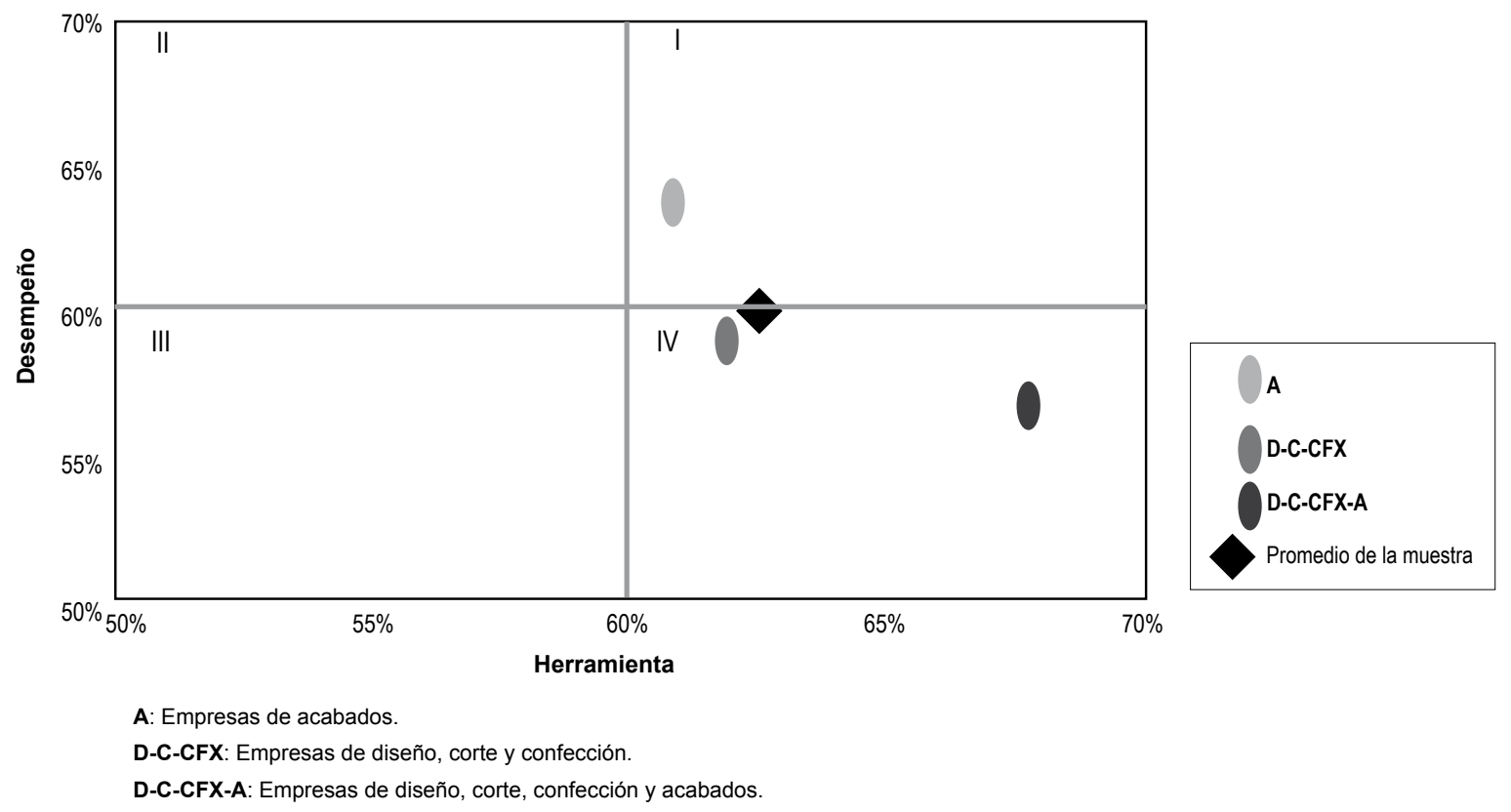

Figura 11. Promedio por proceso productivo, detallado por herramienta y desempeño 


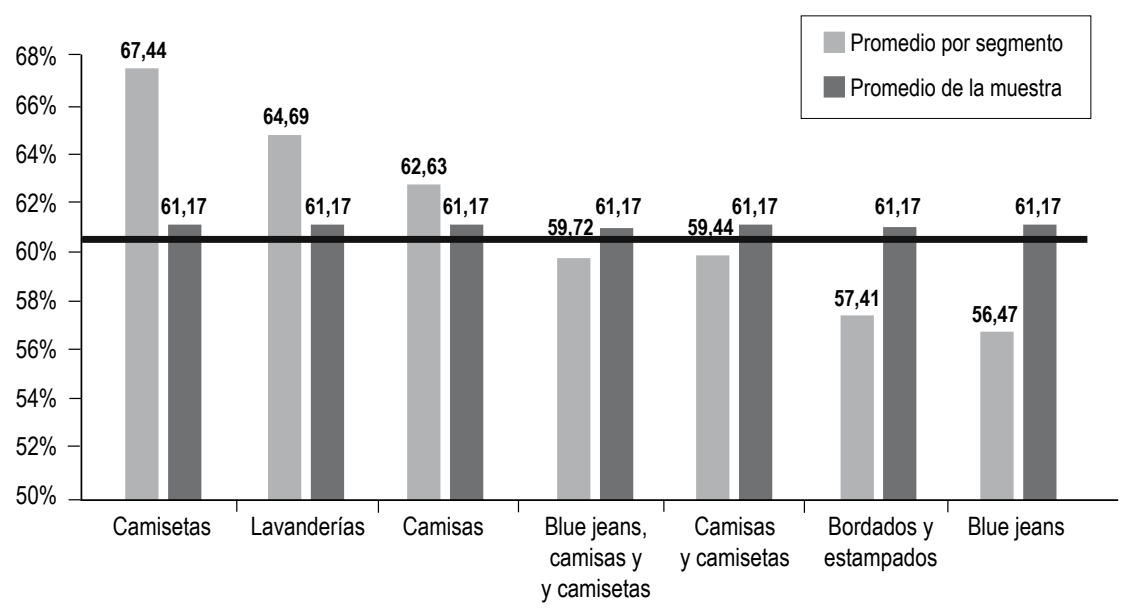

Figura 12. Promedio de cada uno de los segmentos vs promedio general de la muestra

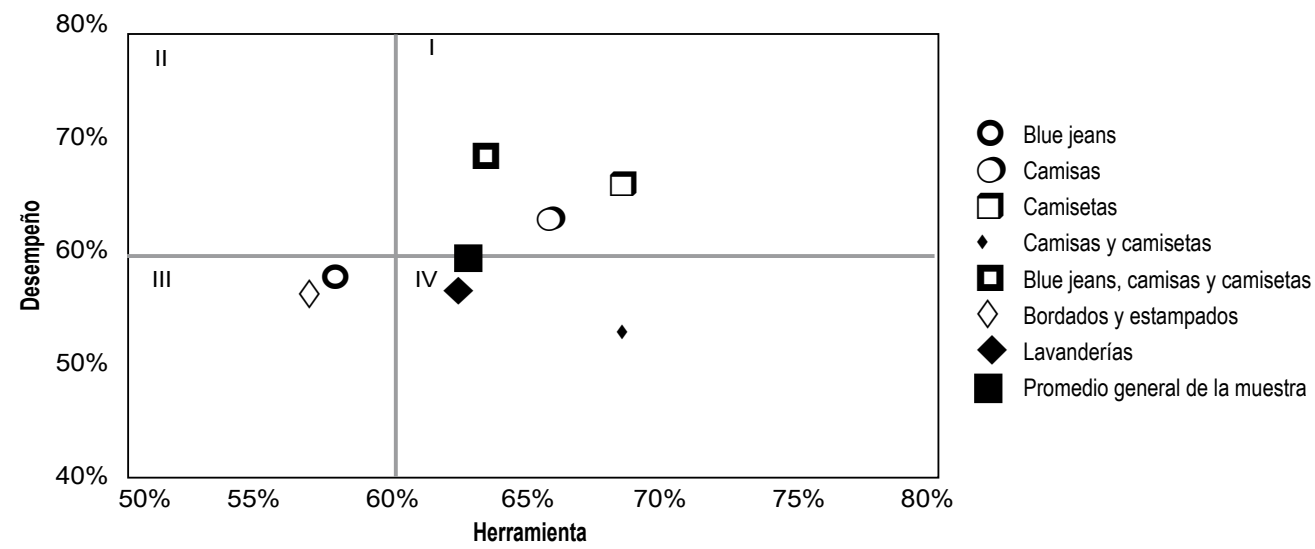

Figura 13. Promedio por segmento, detallado por herramienta y desempeño

\section{Descripción por segmento}

La Figura 12 muestra el promedio de cada uno de los segmentos, comparado con el promedio general de la muestra y el nivel mínimo esperado.

La Figura 13 muestra el promedio de cada segmento por herramienta y desempeño comparado con el promedio general del sector y el nivel mínimo esperado.

\section{Descripción por Política de Producción}

La Figura 14 muestra el promedio por herramienta y desempeño para cada política de producción comparado con el promedio general del sector y el nivel mínimo esperado (MTS: Make to stock/Fabricación para Inventario; MTO: Make to order/Fabricación sobre Pedido; DTO: Design to order/Diseño sobre pedido). 


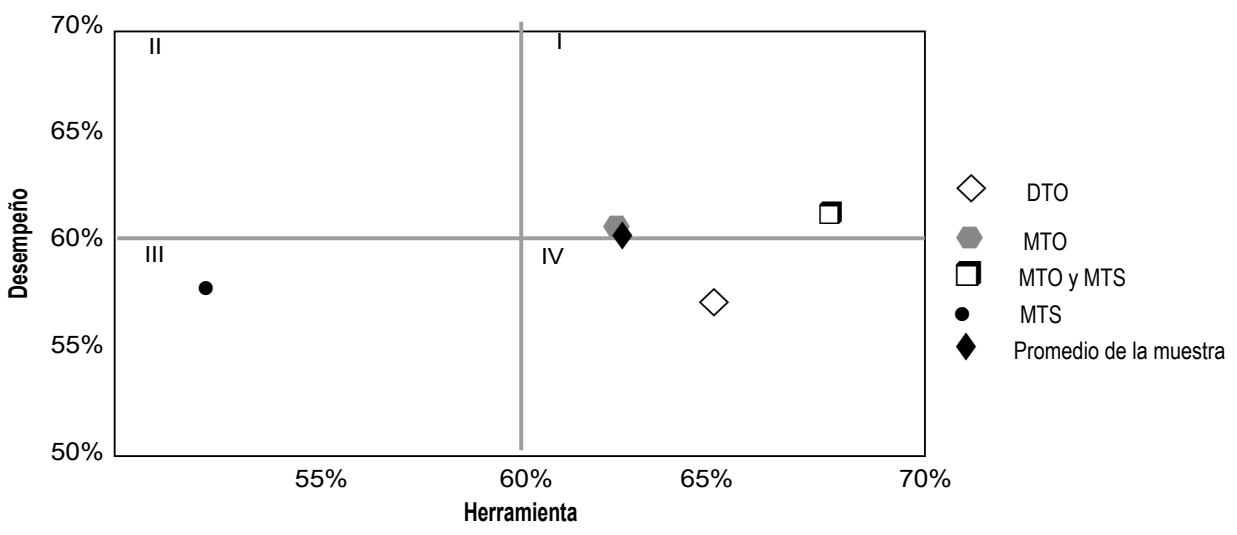

Figura 14. Promedio por empresas según la política de producción

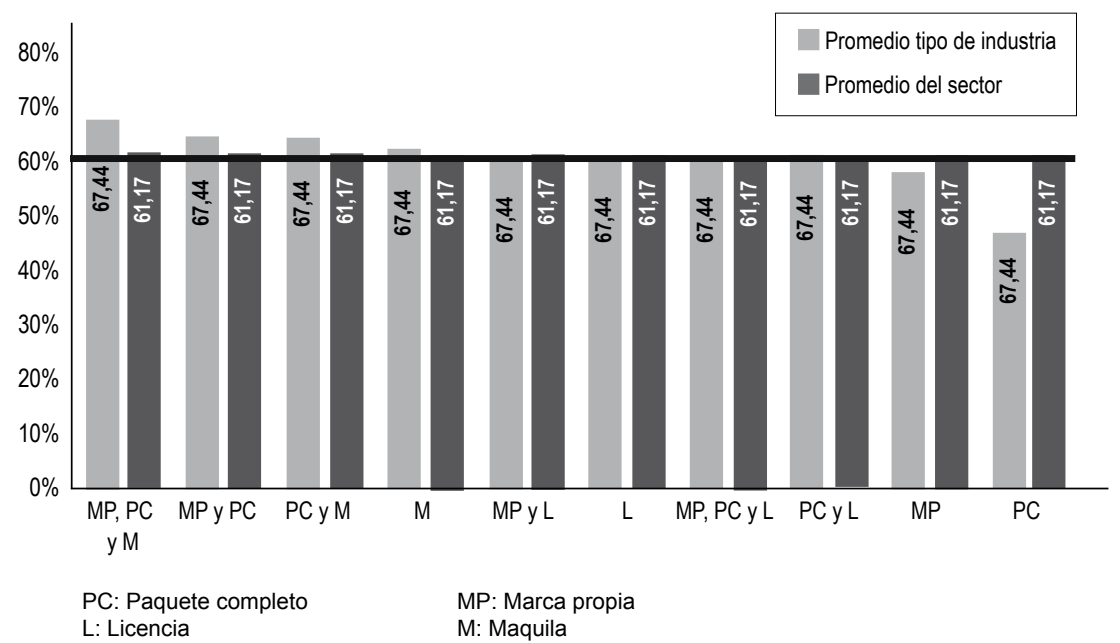

Figura 15. Nivel de cumplimiento de Manufactura Esbelta por tipo de industria

\section{Descripción por Tipo de Industria}

La Figura 15 muestra el promedio de cada agrupación de empresas, según el tipo de industria, comparado con el promedio general del sector y el nivel mínimo esperado.
La Figura 16 muestra el promedio para cada tipo de industria por herramienta y desempeño comparado con el promedio general del sector y el nivel mínimo esperado. 


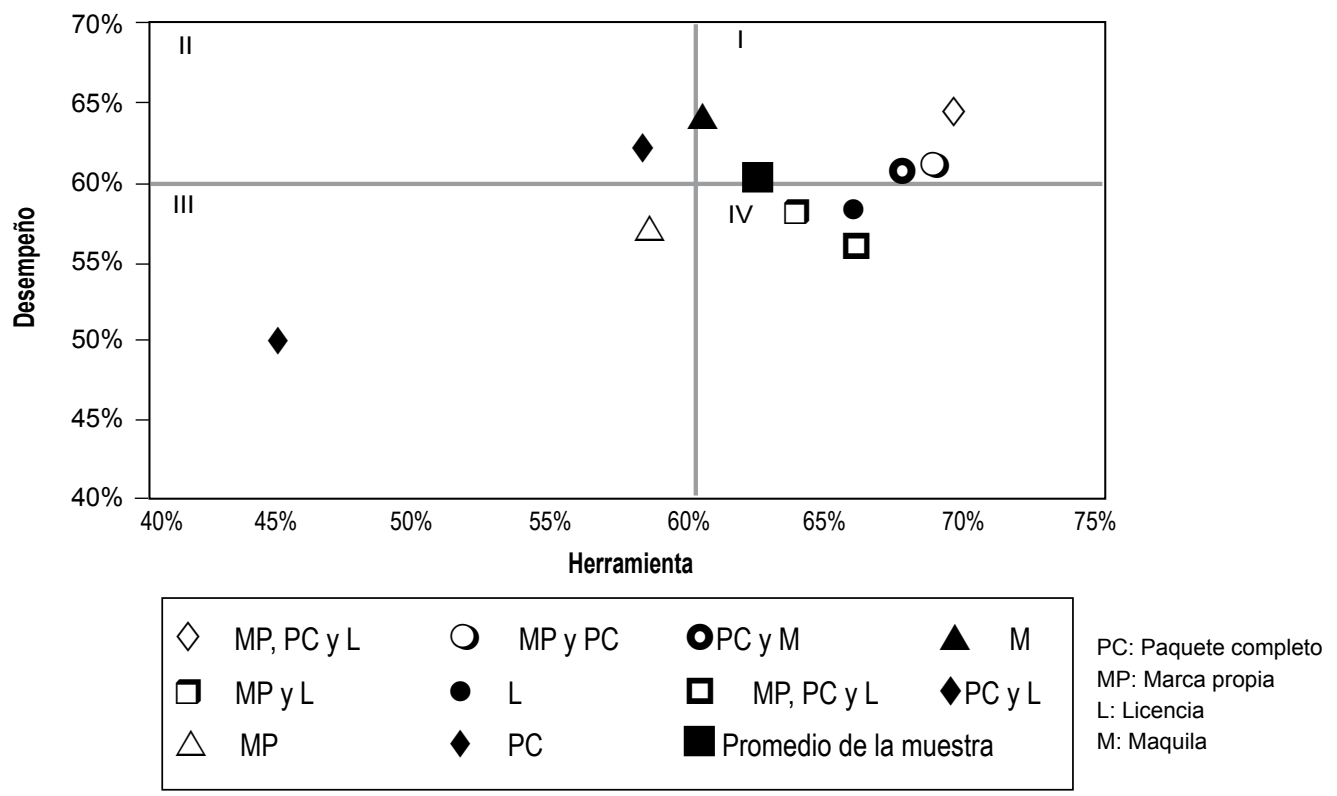

Figura 16. Comparación entre empresas según el tipo de industria

Análisis de correlación entre las variables herramienta y desempeño utilizadas en el estudio

Para determinar si existía dependencia entre las dos variables herramienta y desempeño se aplicó una prueba de hipótesis en la que se busca evaluar si la p (coeficiente de correlación de Pearson) es igual o diferente de cero, teniendo como hipótesis nula ho: $\mathrm{p}=0$, y la hipótesis alternativa $\mathrm{h} 1: \mathrm{p}$ diferente de 0 . Al aplicar la prueba se encontró que el estadístico de prueba $t$ arrojó un valor de $t=3.19$, mayor que el valor crítico de $t$, que arroja un valor de $\mathrm{t}$ crítico $=2.807$. Por lo tanto, se puede afirmar que existe evidencia estadística de que el coeficiente de correlación de Pearson para herramienta y desempeño es diferente de cero; es decir, sí hay dependencia entre las variables con un nivel de confianza del 99\%, la correlación entre el nivel alcanzado en herramienta y en desempeño es positiva, $\left(r=0.517, r^{2}=0.26\right)$ (ver Figura 17). Lo antedicho significa que, a medida que las herramientas se implementan mejor será el desempeño que se logre en la empresa (Ríos, 2006).
La Figura 17 muestra los datos obtenidos de una prueba de dependencia por medio de una regresión lineal con un nivel de confianza del 99\%.

El resultado de este estudio dará pie a nuevos proyectos de mayor envergadura que permitirán generar un modelo de comparación para las empresas del sector con relación a los indicadores de gestión que se deben usar en confección y el nivel en el cual estos deben de estar para ser consideradas empresas de categoría mundial. Adicionalmente, se busca generar las bases para el futuro desarrollo de una plataforma informática apoyada en tecnologías web que permita a las empresas del sector compararse en tiempo real con sus pares en cuanto a la aplicación de las técnicas de manufactura esbelta. Esta herramienta de mejoramiento continuo les ayudará a evaluarse, y así mejorar y aumentar su competitividad

La Figura 4 muestra el promedio general de las 30 empresas evaluadas donde se observa que el total 


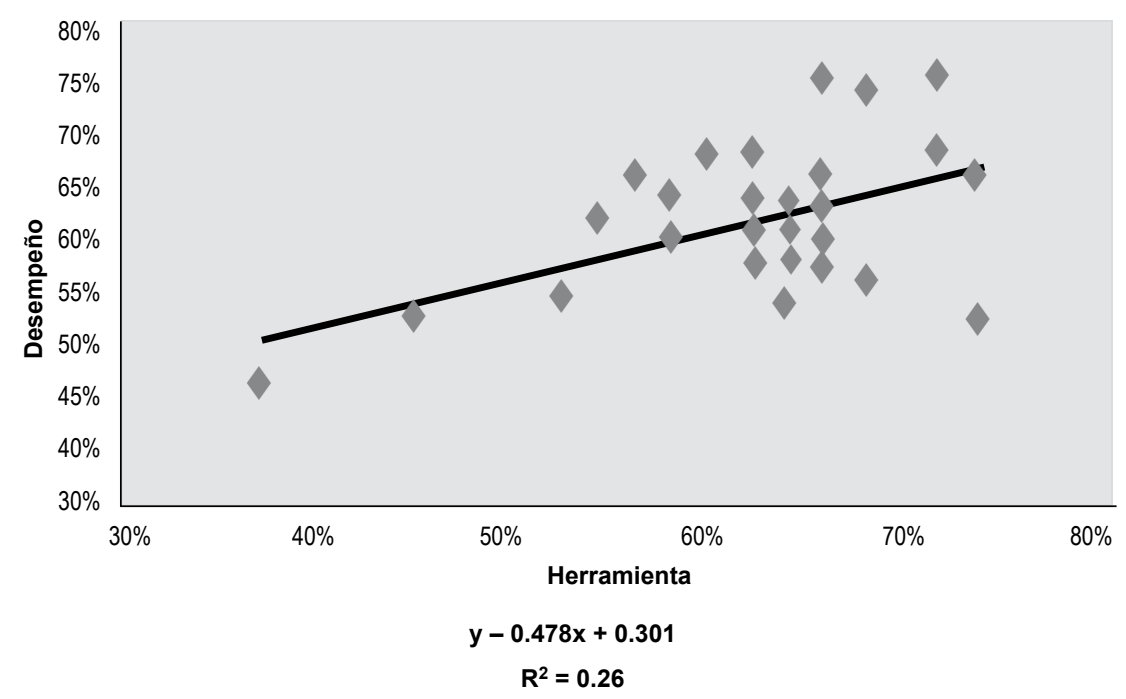

Figura 17. Diagrama de correlación herramienta y desempeño

de la muestra tiene una calificación del $61.17 \%$. Ello indica que la muestra está por encima del valor mínimo esperado (60\%). Por su parte, el cuadrante de la Figura 5 indica el promedio general del total de la muestra, detallado por herramienta y desempeño. Se observa que en la clasificación por herramienta, la muestra se encuentra por encima del valor mínimo esperado, con un resultado del $62.28 \%$, y en desempeño con una calificación inferior pero muy cercana al nivel mínimo esperado del $59.92 \%$. Las cifras indican que el sector no alcanza niveles satisfactorios en la medición del desempeño en prácticas de Manufactura Esbelta; sin embargo, el nivel de cumplimiento en la implementación de las herramientas es bueno.

La Figura 6 presenta dónde se encuentra cada una de las empresas en el cuadrante en el que se evalúa el tipo de compañía con relación a la clase mundial. Es importante resaltar que ninguna de las empresas estudiadas se situó dentro de la calificación que denota ser una empresa de clase mundial y que aplica óptimamente las técnicas de Manufactura Esbelta. Para este caso, las empresas ubicadas en el cuadrante I demuestran ser empresas desafiantes, o sea aquellas cuya evaluación está por encima de $60 \%$ en ambas variables. Por su parte, las empresas en el cuadrante II son vulnerables, es decir, tienen una calificación por encima del $60 \%$ en desempeño, pero inferior al $60 \%$ en herramientas. Las empresas ubicadas en el cuadrante III son empresas contrapeso o que están rezagadas. Ambas calificaciones obtienen cifras inferiores al $60 \%$. Por último, las situadas en el cuadrante IV son empresas promisorias o sea su calificación es mayor al $60 \%$ en herramientas, pero menor a este valor en desempeño. El promedio de todas las empresas se encuentra en el cuadrante IV, donde se ubican las empresas promisorias. Este promedio le confiere al sector confección buenas perspectivas de desarrollo y un muy buen campo de acción para ir mejorando sus condiciones operativas y administrativas de trabajo.

De acuerdo con el cuadrante de empresas de clase mundial, catorce empresas son desafiantes, nueve promisorias, tres vulnerables, dos contrapeso y dos rezagadas. Dicha calificación indica que $76.7 \%$ de las empresas del sector está trabajando correctamente en su orientación hacia las técnicas de mejoramiento de los procesos productivos.

En cuanto a la calificación por herramienta, el 30\% de las empresas evaluadas presentaron niveles menores al mínimo esperado (60\%), y el 50\% arrojaron resulta- 
dos menores al promedio de la muestra en herramienta (62.28\%). Estos porcentajes demuestran que la mitad de las empresas tienen calificaciones mayores al 64\%, y pueden catalogarse en un nivel más competitivo en su gestión de Manufactura Esbelta. En la evaluación de desempeño, $50 \%$ de las empresas se encuentran por debajo del nivel mínimo esperado, y la otra mitad está entre $60 \%$ y $74.7 \%$.

En la Figura 7 se compara la calificación promedio general de cada una de las empresas con el promedio general de la muestra (61.17\%) y con el nivel mínimo esperado (60\%). Como se observó, cuatro de las empresas lideran el sector evaluado, mientras que tres se encuentran muy por debajo del nivel mínimo esperado.

El radar de la Figura 9 detalla y compara el promedio general de cada una de las áreas de trabajo -LMPP (50.40\%), LMPF (66.07\%), LMAC (45.11\%), LMDD $(80.44 \%)$-, con el promedio general de la muestra (61.17\%) y con el nivel mínimo esperado (60\%). Al mismo tiempo, se visualiza el desequilibrio entre las diferentes áreas de trabajo. Adicionalmente se reitera que las áreas de cadena de abastecimiento (LMAC) y Personal (LMPP) no cumplen con el nivel mínimo esperado, mientras que el piso de la fábrica y el diseño, así como el desarrollo de productos están en un buen nivel.

La Figura 10 muestra el comportamiento de los indicadores para el promedio general de todas las empresas evaluadas. El 55\% de los indicadores obtienen un cumplimiento mayor o igual al mínimo esperado; es decir, las empresas están en una proporción aceptable, pero no satisfactoria. Según esta figura, los indicadores LMPF8, 11 y 12 y los indicadores LMDD1 y 5 tienen un cumplimiento mayor al $90 \%$, demostrando que las mayores fortalezas de estas son como sigue:

- Flexibilidad en la producción

- Aplicación de técnicas de balanceo de líneas

- Estandarización de los procesos productivos de la planta

- Aplicación de la ingeniería concurrente o simultánea
- Productos que se fabrican usando módulos de confección.

Los indicadores más deficientes son LMPF3, 4, 9, 13, 15, 17; LMPP1, 2, 5, 6, 8, 9; LMDD3 y LMAC2, $3,4,5$. Estos indicadores presentan promedios menores al $55 \%$, lo que indica que las mayores debilidades son las siguientes:

- Tiempo de entrega (Lead Time) de las órdenes a los clientes

- Entregas justo a tiempo realizadas por los proveedores de la empresa

- Uso de las tarjetas kanban para las actividades de programación de la producción

- Implementación de la fábrica visual

- Implementación del mantenimiento productivo total

- Implementación de sistemas Poka Yoke en la empresa

- Número de sugerencias realizadas por los empleados al año

- Número de sugerencias realizadas por los empleados que son implementadas al año

- Cantidad de horas de entrenamiento por empleado por año en los temas de Lean Manufacturing

- Trabajadores que conocen el tema de Manufactura Esbelta y tienen una visión general del proceso

- Planes de reconocimiento sobre ideas de mejoramiento implementadas

- Aplicación de las $5 \mathrm{~s}$ en sus procesos productivos

- Indicador de porcentaje interno de defectos

- Documentos intercambiados con los proveedores usando EDI

- Cantidad de proveedores certificados que tiene la empresa

- Cantidad de proveedores con acceso al sistema de planeación y control de la producción de la compañía 
- Cantidad de proveedores a los que la empresa tiene acceso a su sistema de planeación y control de la producción

En la Figura 10 también se observan los indicadores que pertenecen a cada una de las áreas de trabajo LMPF, LMPP, LMDD, LMAC respectivamente agrupados y comparados con el nivel mínimo esperado. Se reiteran nuevamente las fortalezas que se presentan en las áreas de piso de fábrica y de diseño, así como el desarrollo del producto en el cumplimiento de Manufactura Esbelta. También están indicadas las debilidades presentes en las áreas de personal y de la cadena de abastecimiento. Notablemente, el área de trabajo que presenta el mejor cumplimiento para la Manufactura Esbelta es la de diseño y desarrollo de productos. En este aspecto, 90\% de las empresas se encuentran por encima del $72 \%$.

En general, a partir de la muestra, se puede concluir que el área de Cadena de Abastecimiento tiene un nivel muy bajo. En este aspecto, el $90 \%$ de los resultados obtenidos están por debajo del $60 \%$ de cumplimiento. Como se observa en la Figura 10, dicha deficiencia está principalmente asociada a los indicadores LMAC2, 3, 4 y 5 , en los cuales las empresas obtuvieron calificaciones menores al $60 \%$. En otras palabras, la mayor debilidad de las empresas se encuentra en la deficiencia durante el intercambio de vía EDI con proveedores, la falta de certificación de proveedores y el acceso al sistema de planeación y control de la producción entre proveedor y empresa en el flujo de información en los dos sentidos.

\section{Análisis según la clasificación de los procesos productivos.}

Las empresas con procesos de diseño, corte, confección y acabados presentan el mayor promedio, $62.50 \%$, seguido por las empresas con procesos de Acabados con el 62.14\%; ambos procesos están por encima del promedio general de la muestra y del nivel esperado. Las empresas con el proceso de diseño, corte y confección $(60.53 \%)$ se encuentran en un nivel inferior al promedio de la muestra, pero superior al $60 \%$.
La Figura 11 muestra el promedio en herramienta y desempeño para cada proceso productivo de las empresas comparándolo con el promedio general del total de la muestra y el mínimo aceptable, $60 \%$. En el cuadrante I, en la categoría desafiante, se encuentran las empresas con el proceso de acabados, y el en IV, calificados como procesos promisorios, se ubican las empresas con los procesos de diseño, corte y confección y de diseño, corte, confección y acabado. Todos los procesos superan el límite mínimo en el área de diseño y desarrollo del producto y en piso de fábrica, mientras que las áreas de personal y de administración de la cadena de abastecimiento aún presentan deficiencias en la implementación de las técnicas de la Manufactura Esbelta.

\section{Análisis según la descripción por segmento}

La Figura 12 muestra el promedio de cada uno de los segmentos comparado con el promedio general de la muestra y el nivel mínimo esperado. Los segmentos de Camisetas, Camisas y Lavanderías se encuentran por encima del promedio general de la muestra y del nivel esperado. Los segmentos restantes se encuentran en un nivel inferior al promedio de la muestra y al valor esperado. El segmento de Blue Jeans es el que presenta una mayor deficiencia.

La Figura 13 muestra el promedio de cada segmento por herramienta y desempeño comparado con el promedio general del sector y el nivel mínimo esperado. En el cuadrante I, calificado como desafiantes, se encuentran los segmentos de Camisetas, Camisas y Lavanderías. En el cuadrante III, se ubican Blue Jeans y BordadosEstampados como segmentos de contrapeso. En el IV, como provisorios, están Blue Jeans-Camisas-Camisetas y Camisas-Camisetas.

Los segmentos estudiados presentan muy buen cumplimiento en la aplicación de las técnicas de Manufactura Esbelta en las áreas de diseño y desarrollo del producto y de piso de fábrica; en tanto, las cadenas de abastecimiento y personal muestran debilidades. 


\section{Análisis según la política de producción}

Los mayores promedios los obtienen las empresas que combinan MTO y MTS (fabricación para inventario y fabricación sobre pedido), y las que son únicamente MTO, (fabricación sobre pedido). Ambas categorías observan, como es de esperarse, un cumplimiento incluso mayor al del promedio general. Por otra parte, el promedio mínimo es de las empresas MTS (fabricación para inventario) que arrojan un promedio menor al $60 \%$.

La Figura 14 muestra el promedio para cada política de producción por herramienta y desempeño comparado con el promedio general del sector y el nivel mínimo esperado. En la calificación de desafiantes del Cuadrante I se encuentran las empresas MTO y aquellas que simultáneamente implementan MTS y MTO; sin embargo, los niveles obtenidos en desempeño son bajos. En el cuadrante III, como empresas de contrapeso, se encuentran las firmas MTS que por su estilo de gestión contraria a la Manufactura Esbelta obtuvieron niveles muy bajos tanto en el aspecto herramienta como desempeño. En general, las empresas en esta clasificación se encuentran principalmente por debajo del eje longitudinal; es decir, en general los niveles de desempeño no son buenos.

\section{Análisis según el tipo de industria}

El sector en general tiene un comportamiento que oscila muy cerca del valor mínimo esperado y del promedio del sector; en otras palabras, las empresas ostentan un nivel aceptable, pero no satisfactorio (ver Figura 15). La mayor calificación corresponde a las empresas que trabajan con marca propia, paquete completo y maquila simultáneamente; ellas alcanzan un puntaje del $67 \%$, mientras que las industrias de marca propia y paquete completo están en niveles de cumplimientos menores al $60 \%$.

La Figura 16 demuestra que los puntajes para herramienta y desempeño están muy equilibrados para los diferentes tipos. Sin embargo, los niveles alcanzados por las herramientas son mayores. El tipo de industria MP-PC-M presenta la mayor calificación y se ubica en el primer cuadrante como desafiante. Esta industria tiene un buen desempeño e implementación de las herramientas Lean, mientras que las de Paquete Completo (PC) se califican como rezagadas, pues no logran buenos niveles en el desempeño e implementación del Lean Manufacturing.

\section{Acciones para el mejoramiento}

En esta sección se presentan de manera concreta las acciones de mejoramiento por cada una de las áreas de evaluación que las empresas deben emprender para incrementar su calificación dentro del sector e ir acercándose a las características de una empresa de categoría mundial. Estas acciones de mejora son las siguientes:

1. Área de personal (LMPP): Implementar planes de sugerencias y el montaje del programa $5 \mathrm{~s}$, así como en la formación del personal en las diferentes técnicas para el mejoramiento de los procesos productivos.

2. Área de la administración de la cadena de abastecimiento (LMAC): Buscar asesoría en institutos especializados en el manejo de las Tics, y empezar a desarrollar proyectos de estandarización de tareas administrativas dentro de la empresa y con los proveedores y clientes que los lleven a un montaje posterior de sistemas de intercambio electrónico de datos entre todos los actores de la cadena.

3. Área de diseño y desarrollo de productos (LMDD): Implementar mecanismos Poka Yoke y desarrollar planes de control de calidad basados en técnicas estadísticas, en paralelo con la formación del personal con la aplicación de estas.

4. Área del piso de la fábrica (LMPF): Implementar programas de mantenimiento productivo total, TPM, empezando con la capacitación del personal en los temas de mantenimiento autónomo y $5 \mathrm{~s}$.

Recomendaciones generales para cada una de las áreas de trabajo

En la Tabla 3 se presentan de manera más objetiva las acciones de mejoramiento que las empresas requie- 
Tabla 3.

Recomendaciones para cada una de las áreas de trabajo

\begin{tabular}{|c|c|}
\hline Área de Trabajo & Recomendaciones \\
\hline LMPF & $\begin{array}{l}\text { Implementar programas de mantenimiento productivo total, TPM, empezando con la capacitación del } \\
\text { personal en los temas de mantenimiento autónomo y } 5 \mathrm{~s} \text {. }\end{array}$ \\
\hline LMPP & Implementar planes de sugerencias y el montaje del programa $5 \mathrm{~s}$, también la formación del personal. \\
\hline LMDD & $\begin{array}{l}\text { Implementar mecanismos Poka Yoke y desarrollar planes de control de calidad basados en técnicas } \\
\text { estadísticas, en paralelo con la formación del personal con la aplicación de estas. }\end{array}$ \\
\hline LMAC & $\begin{array}{l}\text { Buscar asesoría en institutos especializados en el manejo de las Tics, y empezar al interior de la empresa } \\
\text { y con los proveedores y clientes a desarrollar proyectos de estandarización de tareas administrativas } \\
\text { que lleven a un montaje posterior de sistemas de intercambio electrónico de datos entre todos ellos. }\end{array}$ \\
\hline
\end{tabular}

ren emprender cada una de las áreas de evaluación para incrementar su calificación dentro del sector e ir acercándose a las características de una empresa de categoría mundial.

\section{CONCLUSIONES}

Después de la aplicación del modelo de Spendolini a través del cuestionario desarrollado para diagnosticar el estado de Manufactura Esbelta en el sector de la confección en la ciudad de Medellín, específicamente para los segmentos de Blue Jeans, camisas Tipo Polo y $t$-shirts, se encontró que para las 30 empresas de la muestra la calificación general promedio es de $61.17 \%$. Este resultado está por encima del nivel mínimo aceptable; sin embargo, es muy deficiente al compararlo con el de empresas de categoría mundial. Ninguna de las empresas evaluadas se encuentra en los niveles superiores del primer cuadrante, con resultados por encima del $80 \%$.

En el análisis del cuadrante, el sector analizado se ubica entre las empresas promisorias, $(62.28 \%$ y $59.92 \%$ ), lo que significa que tienen implementadas técnicas de mejoramiento y están a la espera de conseguir resultados o mejorar los existentes. Esto, de manera clara, responde a la pregunta de hipótesis establecida al inicio del estudio de que sí es posible evaluar el grado de implementación de las técnicas de Manufactura Esbelta en el sector confección.
La implementación de herramientas de Manufactura Esbelta se encuentra en un $62.28 \%$ de implementación, valor menor al nivel de categoría mundial. Las empresas evaluadas no han desarrollado la cultura ni han implementado de manera consciente e integral la filosofía Lean Manufacturing; es decir, son empresas en que las condiciones de producción del mercado y de búsqueda de competitividad las han llevado a implementar las herramientas mínimas que les permite llegar a este nivel. En el estudio se encontró que las herramientas menos implementadas son:

- Uso de plataformas para el intercambio electrónico de datos entre las empresas y sus proveedores;

- certificación de proveedores;

- uso de tarjetas kanban;

- implementación 5'S.

El nivel obtenido por las empresas en la variable Desempeño es del 59.92\%. La cifra refleja la insuficiencia con que es llevada a cabo la medición en los procesos productivos. Las mayores debilidades encontradas fueron:

- falta de capacitación y conocimiento en temas de mejoramiento para los operarios especializados y de planta;

- deficiencia en el sistema de sugerencias por falta de implementación y compromiso de los empleados; 
- alta tasa de productos defectuosos;

- falta de oportunidad en los tiempos de entrega justo a tiempo.

Del análisis realizado se puede observar que la mayoría de empresas (57\%) están en los cuadrantes I y IV, como empresas promisorias o desafiantes, lo que indica que el sector en general está aplicando tareas de mejoramiento, aunque están localizadas en el límite inferior, o mínimo deseable. Las empresas de confección son las que mejor se encuentran en la aplicación de la Manufactura Esbelta, pues obtiene el $71.43 \%$ de empresas desafiantes. También se debe a que alcanzan un nivel de cumplimiento promedio del $64.9 \%$, valor que se encuentra por encima de los resultados obtenidos por las lavanderías y las bordadoras-estampadoras.

Por otro lado, se encontró que el tema de Manufactura Esbelta está desligado de las estrategias del negocio. Es decir, las iniciativas no surgen desde la alta gerencia ni de accionistas, sino de los mandos medios que proponen pequeñas aplicaciones acorde con las necesidades, y que estos conocimientos no son transmitidos a los niveles operativos. Dicho lo anterior, se concluye que los temas de Manufactura Esbelta para el sector no están enlazados dentro de los modelos de gestión de las organizaciones porque no encajan dentro de las medidas de desempeño y control de la cotidianeidad del proceso y, por ende, tampoco en la parte táctica y estratégica de las empresas.

Los resultados muestran que el sector confección en general tiene su mayor fortaleza, analizado desde las técnicas de Manufactura Esbelta, en el área de Diseño y Desarrollo de Productos, con un promedio de $80.44 \%$, comparándose con empresas de categoría mundial. Esta área es un factor clave de éxito que les permite ser competitivos y diferenciarse en el mercado, pues presentan altos niveles de flexibilidad y variedad de productos. Sin embargo, el sector de la confección aún tiene muchos puntos débiles. Uno de los más importantes es el porcentaje de productos defectuosos, LMDD3 (38.7\%). Esta cifra indica que existen falencias en la aplicación de dispositivos Poka Yokes (LMPF 17, $37.3 \%$ ) que garanticen la calidad en la fuente y en el control estadístico de calidad (LMPF 16, 61.3\%). Este aspecto es muy significativo, porque lo que no se mide, no se puede controlar y no se puede mejorar.

El área de Piso de la Fábrica, en la que se administra al personal operativo, los flujos de producción, la planificación y control de la producción, así como las tareas de mantenimiento, se encuentran en segundo nivel para el sector. No obstante, no llega a niveles satisfactorios puesto que el promedio está más cerca del $60 \%$ que del $80 \%$. El cumplimiento obtenido en herramienta refleja que las técnicas Lean se están implementando; sin embargo, los niveles de desempeño son bajos porque la implementación de las herramientas es incipiente. Ello significa que no llevan el tiempo suficiente para estar maduras y arrojar altos niveles de desempeño, y así alcanzar los niveles de las empresas desafiantes.

El sector de la confección tiene una gran deficiencia en el Área de Personal. El aspecto cultural para soportar la implementación y garantizar la permanencia de Manufactura Esbelta se encuentra por debajo del mínimo esperado (50.4\%). Esta baja calificación se debe a la falta de participación y compromiso por parte de los operarios y directivos de la empresa en las diferentes actividades de mejoramiento y a la formación con respecto de estos temas.

El Área de Administración de la Cadena de Abastecimiento es la que requiere generar planes de mejoramiento mucho más definitivos y concretos. Esta es la que menor valoración alcanzó en el estudio con $45.11 \%$. La carencia de tecnologías de información y comunicaciones para la administración del negocio, y la relación con los proveedores y clientes, hace que este grupo de preguntas obtuviera la menor calificación.

En el cuadrante I se encuentran 13 empresas que son las que mejores resultados obtuvieron. En general, ellas son las que aplican técnicas de Manufactura Esbelta y presentan buenos niveles de desempeño gracias a la estructura de las empresas, sus políticas, su relación con proveedores y clientes. Además, han desarrollado y continúan con la implementación de técnicas de mejo- 
ramiento. Sin embargo, a pesar de ser calificadas como desafiantes, están más cerca de los límites del $60 \%$ que del $80 \%$. El segmento con mejores promedios es el de Camisetas, quizás porque la producción está enfocada a un solo producto. Por lo tanto, el personal está especializado en el producto, se tiene un mejor control estadístico y de calidad facilitando la estandarización de los productos y procesos. También, son empresas que poseen máquinas específicas y generales que les permiten ser flexibles.

El segmento de Blue Jeans presenta los menores promedios por herramienta y por desempeño, ubicándose por debajo de los niveles mínimos en el tercer cuadrante. Por tal motivo, son empresas calificadas como de contrapeso. Esto significa que les falta implementación y capacitación de herramientas de Manufactura Esbelta; deben mejorar los puntos más débiles que presentaron, tales como la flexibilidad para responder a cambios imprevistos de la demanda, el número de productos defectuosos y la capacitación en los temas de Manufactura Esbelta en operarios especializados y de planta.

Las empresas del proceso Diseño-Corte-Confección y Acabados presentaron el mejor promedio ante los otros procesos, incluso mayor al promedio general del sector. La razón de este resultado es que tienen un mayor dominio del proceso productivo gracias a la integración vertical. Sin embargo, todas las empresas de este grupo están calificadas como promisorias porque tienen una satisfactoria implementación de herramientas, pero no llegan a un buen nivel de desempeño.

Como es de esperar, las empresas que se rigen por la política de producción MTO (fabricación sobre pedido) puro, o combinado con MTS, (fabricación para inventario) alcanzan mayores niveles de cumplimiento, porque son coherentes con el principio de justo a tiempo de la filosofía estudiada. Mientras, por otro lado, las empresas MTS puras están en el nivel de rezagadas, porque la dinámica de sus políticas de producción se opone a los principios de la Manufactura Esbelta. Debido a la correlación que existe entre herramientas y desempeño, es posible mejorar los resultados obtenidos al implementar acciones de mejora, si estos apuntan, en primera medida, a elevar la calificación de los indicadores de herramienta, de esta manera directamente se aumentarían los de desempeño.

El sector confección no tiene bien implementadas las técnicas de mejoramiento de procesos basadas en la Manufactura Esbelta. Ello se debe a las problemáticas que se debaten a nivel político y económico; no solo en el ámbito nacional, sino también en el mundial. Esta situación se hace especialmente más aguda por la caída en la demanda y el contrabando. Por otro lado, se debe tomar en cuenta que el sector es muy dependiente de la mano de obra operativa y no existe una fidelidad a la empresa por parte de los operarios. Esto significa que existe una alta rotación de personal, lo que conlleva a que dichos procesos de mejora no perduren en el tiempo o sean más difíciles de implementar. También se observa que las directivas de las empresas buscan resultados a corto plazo, y la implementación de estas técnicas no da resultados inmediatos.

\section{Proyectos futuros}

A partir de la estructura de este proyecto, las empresas estudiadas y, especialmente, las características evaluadas, se pueden conducir nuevos estudios más detallados sobre el mismo sector de la confección en el que se evalúen los beneficios en productividad, polivalencia, calidad y eficiencia al implementar los módulos de confección en las diferentes empresas. Igualmente, se puede ampliar este estudio utilizando los mismos indicadores y cuestionario de Manufactura Esbelta a otros sectores industriales y también el de la confección, industria de gran importancia en la ciudad de Medellín, especialmente el de la ropa interior femenina y masculina y los vestidos de baño, y que generalmente utilizan la misma tecnología y características de la mano de obra. Por otro lado, y de acuerdo con uno de los resultados que arrojó este proyecto, sería muy interesante conducir un estudio en el que se puede evaluar y analizar el estado en el que se encuentra el sector de confección en cuanto a la aplicación y conocimiento de las Tics (tecnologías de información y comunicaciones) para la administración y control de la producción y la administración de la cadena de abastecimiento. 
Es importante, también, realizar trabajos en otras áreas fuera del proceso productivo analizado. Por ejemplo, se podrían desarrollar investigaciones sobre la cadena de abastecimiento de las empresas estudiadas en el sector, evaluando, los tiempos de entrega, la forma del empaque y embalaje, la calidad del servicio, el cumplimiento, la forma de almacenamiento y la rotación de los inventarios. Un estudio de esta naturaleza podría tomar los trabajos presentados por el profesor E. H. Frazelle en su libro World Class Warehousing and Material Handling (2002), donde se desarrollan los lineamientos para hacer un benchmarking sobre el almacenamiento de clase mundial.

\section{Recomendaciones}

- Para estudios futuros, se sugiere, a partir de estos resultados obtenidos, incluir indicadores cuantitativos con datos reales de las empresas tales como puede ser la medición del servicio al cliente.

- Es importante que las empresas, sobre todo aquellas que están en el cuadrante III, es decir, empresas clasificadas como rezagadas o de contrapeso, empiecen inmediatamente a desarrollar planes de mejoramiento basados en técnicas de Lean Manufacturing. Se recomienda que inicien su mejoramiento con las 5 s como técnica de control del proceso productivo y administrativo por su gran aplicabilidad y facilidad de capacitación al personal.

- Para las preguntas del área LMAC (administración de la cadena de abastecimiento) que obtuvo las más bajas calificaciones, las empresas deben buscar asesoría de institutos especializados en el manejo de Tics. Es más, estas empresas deben empezar a desarrollar proyectos al interior de sí mismas que propendan la automatización de la información, tanto propia como la que comparten con los clientes y proveedores. El manejo de la información es vital para una buena cadena de abastecimiento.

- De acuerdo con los resultados deficientes en el área de trabajo Personal, se recomienda que el sistema de sugerencias sea respaldado por una cultura de mejoramiento que le permita hacer buen uso de este.

- En el área de Diseño y Desarrollo de Productos se recomienda iniciar un plan de acción que permita reducir el porcentaje de productos defectuosos, incrementando el control de calidad autónomo. Esto significa que cada operario garantice que no se agregue valor a prendas que vienen defectuosas, que no se generen en su operación y, mucho menos, que pasen a una estación posterior. Adicionalmente, se debe apalancar el control de calidad con la aplicación de dispositivos Poka Yokes que garanticen la calidad en la fuente. Asimismo, el control estadístico de calidad debe medir constantemente la producción para mantener los defectos controlados y mejorar los resultados de acuerdo con los datos recolectados.

- Es necesario que la alta gerencia decida incluir la filosofía Manufactura Esbelta como estrategia de competitividad. Para tal efecto, deberá conectar el nivel táctico y operativo, incluyendo los indicadores Lean, dentro del sistema de medición de la compañía, de acuerdo con la estrategia de manufactura que tenga la empresa (costo, calidad, tiempo de entrega y/o flexibilidad). Este aspecto es importante para generar una cultura de mejoramiento continuo.

- Debido a las falencias y debilidades que muestra el sector en el área de Piso de Fábrica y de Personal, y la falta de cultura de mejoramiento continuo, es necesario que el sector inicie la tarea de implementación de las herramientas de producción como son los sistemas visuales, los sistemas Poka Yokes, el uso de tarjetas kanban, la implementación de los pilares del Mantenimiento Productivo Total y la aplicación de $5 \mathrm{~s}$ en sus procesos productivos.

- El sector de la confección tiene oportunidades de mejora muy grandes si promueve entre sus proveedores de insumos a cumplir con los plazos acordados; es decir, que se eliminen las recepciones, 
los chequeos, el almacenamiento y el papeleo, así como que se realicen negociaciones por mes, y que solo se licite para artículos nuevos. Para ello es necesario que se seleccione un único proveedor por tipo de artículo, preferiblemente de la zona, con que ofrezca calidad certificada para mantener relaciones a largo plazo y que realice entregas diarias en poca cantidad, según la necesidad, directamente en la línea.

- Se observó que el Lead Time (tiempo de entrega) establecido en el cuestionario no es concurrente con la realidad del sector. Los resultados obtenidos fueron deficientes, como lo demuestra la calificación de 40\% del indicador LMPF3. Por lo tanto, se recomienda seleccionar el Lead Time como punto de evaluación y comparación para las empresas del sector. Adicionalmente, se sugiere que las empresas revisen el proceso para recortar tiempos de desplazamiento, tiempos de cola, tiempo de cambio de referencia con técnicas SMED y ayudarse con la implementación de tarjetas kanban que hagan un efecto Pull, con el fin de reducir el tiempo de entrega a los clientes.

- Como factor de éxito, los empresarios del sector deben enfocarse en mercados y productos con mayor valor agregado. Para ello, se debe tener una aspiración sectorial clara y trabajar como cadena para alcanzar sus objetivos. Como factor de riegos, hace falta motivar a los empresarios para invertir, transformarse y cooperar entre sí.

\section{Referencias}

Arrieta, J. G. (2007). Interacción y conexiones entre las técnicas 5s, SMED y Poka Yoke en procesos de mejoramiento continuo. Tecnura, 20(1st Semestre), 139-148.

Bheda, R, Narag., A. S, \& Singla, M. L. (2003). Apparel Manufacturing a Strategy for Manufacturing Improvement. Journal of Fashion Marketing and Management, 7(1), 12-22.

Bilalis N, Alvizos, E., Tsironis., L, \& Van Wasseenhove, L. (2007). Benchmarking the competitiveness of industrial sectors. International Journal of Productivity and Performance Management, 56(7), 603-622.

Bolisani, E, \& Scarso, E. (1996). International Manufacturing Strategies: Experiences from the Clothing Industry. International Journal of Operations and Production Management, 16(11), 71-77.

Botero, L. F., \& Álvarez, M. E. (2006). Bench-Colombia: sistema de referenciación para la construcción. (1 ra. Ed.). Medellín, Colombia. Centro de Publicaciones Universidad EAFIT.

Clare, L., \& Mathaisel, D. (2005). An Exploratory Analysis in Applying Lean Manufacturing to a Labor Intensive Industry in China. Asia Pacific Journal of Marketing and Logistics, 17(4) 63-80.
Collins, R., Cordon, C., \& Julien, D. (1996). Lessons from "Made in Switzerland Study" What Makes a World Class Manufacturer. European Management Journal, 14(6), 576-589.

Dickerson, K. (1995). Textiles and Apparel in the Global Economy. New York: Prentice Hall.

Frazelle. E. H. (2002). World Class Warehousing and Material Handling. Nueva York: McGraw Hill.

GS1 < http://www.gs1co.org/>. Recuperado el 29 de mayo de 2008 .

Goodman, R. E. (2002). Read a Plant- Fast. Harvard Business Review, 80(5), 105-113.

Instituto para la Exportación de la Moda - Inexmoda (2009). Recuperado el 25 de octubre de 2009 de <www. inexmoda.org.co $>$.

La Nota Económica (Enero 2006). Textiles y Confecciones: Estudio Platinum. Bogotá, Colombia. Recuperado el 26 de octubre de 2009, <http://lanota.com/index. php/CONFIDENCIAS/Ranking-confeccionistas-deColombia.html>.

Manotas, D. F., \& Rivera, L. (2007). Lean Measurement: The Relationship between Activities and Lean Metrics. Revista Estudios Gerenciales, 23, 105-115. 
Martínez, A., Pérez, M. (2001). Lean Indicators and Manufacturing Strategies. International Journal of Operations \& Production Management, 21, 148149.

Méndez, J. F. (2005). Desenvolvimento de uma ferramenta para operacionalizar o estudo de benchmarking made in Brazil. Dissertação de mestrado (Pós graduação em engenharia de produção). Florianópolis, Brasil: Universidade federal de Santa Catarina.

Navactiva <http:/www.navactiva.com/web/es/alog/doc/ informes/2006/03/36349.php>. Recuperado el 29 de mayo de 2008.

Osorio, A., Restrepo, A., \& Velandia, M. (2001). Estudio de las mejores prácticas en manufactura conocidas como herramientas de producción aplicadas en el sector metalmecánica de la ciudad de Medellín. Trabajo de grado (Ingeniería de Producción). Medellín, Colombia: Universidad EAFIT.

Pereira, G. J. (2006). Um Método de diagnóstico do potencial de aplicação da manufactura enxuta na indústria têxtil. Tese de Pós graduação em engenharia de produção. Florianópolis, Brasil: Universidade Federal de Santa Catarina.

Revista Dinero (junio 2009). < http://www.dinero.com/negocios/busca-oportunidades_62098.aspx $>$. Recuperado el 25 de octubre de 2009.
Ríos, J. G. (2006). Métodos estadísticos. Notas guías de clase. Monterrey, México: Instituto Tecnológico y de Estudios Superiores de Monterrey.

Romero, J., Sandrea, M., Morales, M., Boscán, M., \& Acosta, A. (2000). La industria de la confección zuliana en la era de la competitividad. Revista Venezolana de Gerencia, 5(11), 189-208.

Seibel, S. (2004). Desarrollo de un modelo de benchmarking basado en un sistema productivo de clase mundial para evaluación de prácticas y desempeño de la industria exportadora brasilera. Tesis Doctorar en Ingeniería. Florianópolis, Brasil: Universidade Federal de Santa Catarina.

Silva Rueda, J. (2003). Manufactura esbelta una nueva alternativa de mejoramiento de proceso de producción con conocidas herramientas de ingeniería industrial. Ingeniería y Universidad, 7(2), 193-203.

Spendolini, M. J. (1994). Benchmarking. Bogotá, Colombia: Editorial Norma.

Ungan, M. (2007). Manufacturing Best Practices. Implementation Success Factors and Performance. Journal of Manufacturing Technology and Management, 18(3), 333-348.

Womack, J., Jones, D. T., \& Roos, D. (1996). Lean Thinking: Banish Waste and Create a Wealth in your Corporation. New York: Simon and Schuster. 


\section{INSTRUCTIONS FOR AUTHORS}

- The articles should be unpublished material that may be written in English or Spanish, and they should be accompanied by a brief résumé from the author.

- Only scientific papers will be published, such as theoretical works (conceptual development or critical analysis), methodological application or empirical research.

- The length is open, but the minimum acceptable is 6,300 words.

- All contributions undergo a peer-reviewed process. There are at least two reviewers per article. To guarantee impartiality of the evaluation, the arbiters do not know the identity of the author or authors.

- The first page of the article should contain title, summary and keywords of the article both in English and Spanish, as well as the following information of the author/authors: first name (one only) and last name, institutional affiliation (indicating the country), the highest degree achieved (indicating the university where it was obtained) and the institutional mail address.

- The paper must start with a brief summary of the content (between 125 and 210 words), in which it is specified the author's/authors' scientific contribution. The summary should be followed by between three and five keywords that will allow identifying the subject of the paper.

- In general, the paper must have the following: introduction, theoretical framework, methodology, development of the work (which would highlight the author's/authors' scientific contribution), conclusions and/or recommendations, and complete bibliographic references.

- Tables and figures are numbered sequentially. The titles of the tables are located on the top, while the titles of the figures are located underneath. Figures are graphics, maps, diagrams, photographs, and all kind of illustrations.

- Taking into account that the Journal is published in black and white, the lines, bars and other graphic elements should differ using only black, white and varying degrees of gray.

- The bibliographic references section should only include the complete information of the authors specifically mentioned in simplified form in the body of the paper (author, date: pages).

- Footnotes should be numbered sequentially and employed only to give explanation or more information; they should not contain bibliographic references.

- The bibliographic reference section uses the APA (American Psychological Association) style. For more information on this format, please refer to the following electronic address: $<$ http://owl.english.purdue.edu/owl/ resource $/ 560 / 01 />$.

- Journal contributions should be directed to the Vicerrectorado de Investigación de la Universidad ESAN, and the electronic file should be sent to <esanediciones@esan.edu.pe>. Also, compact discs may be mailed to the Vicerrectorado de Investigación de la Universidad ESAN, Alonso de Molina 1652, Surco, Casilla Postal 1846, Lima 100, Peru. 